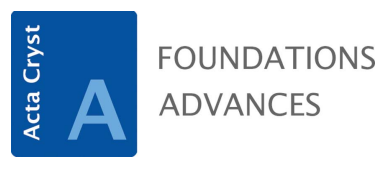

ISSN 2053-2733

Received 12 January 2017

Accepted 10 April 2017

Edited by W. F. Kuhs, Georg-August University Göttingen, Germany

Keywords: thermoelectricity; transport properties; magnetic order; galvanomagnetic effects; thermomagnetic effects.

\begin{tabular}{|c|c|c|c|}
\hline $\begin{array}{l}\text { Magnetic } \\
\text { form classes }\end{array}$ & & $\begin{array}{l}k_{i j}^{0-} \\
\rho_{i j}^{0-}\end{array}$ & $\Sigma_{i j}^{0-}$ \\
\hline & $\begin{array}{l}i \\
\downarrow\end{array}$ & $\begin{array}{ccc} & j \downarrow & \\
& & \\
1 & 2 & 3\end{array}$ & $\begin{array}{ccc} & j \downarrow \\
1 & 2 & 3\end{array}$ \\
\hline 1 & 1 & o & $\begin{array}{lll}\bullet & \bullet & \bullet \\
\bullet & \bullet & \bullet \\
\bullet & \bullet & \bullet\end{array}$ \\
\hline
\end{tabular}

C 2017 International Union of Crystallography

\section{Thermoelectric transport properties in magnetically ordered crystals}

\author{
Hans Grimmer* \\ Research with Neutrons and Muons, Paul Scherrer Institut, WLGA/019, Villigen PSI, CH-5232, Switzerland. \\ *Correspondence e-mail: hans.grimmer@psi.ch
}

The forms of the tensors describing thermoelectric transport properties in magnetically ordered crystals are given for frequently used orientations of the 122 space-time point groups up to second order in an applied magnetic field. It is shown which forms are interchanged for the point groups of the hexagonal crystal family by two different conventions for the connection between the Hermann-Mauguin symbol and the orientation of the Cartesian coordinate system. The forms are given in Nye notation, which conspicuously shows how the forms for different point groups are related. It is shown that the measurable effects in magnetically ordered crystals can be decomposed into an effect occurring in all crystals and one coming from the magnetic ordering. Errors in the literature are pointed out.

\section{Introduction}

Based on general principles of statistical physics (Landau \& Lifshitz, 1958) Shtrikman \& Thomas $(1965 a, b)$ gave the Onsager relations in a form convenient for deriving transport properties in magnetically ordered crystals. They applied the relations to the electric resistivity of crystals in an external magnetic field $\mathbf{H}$. Similar results were also obtained by Butzal \& Birss (1982), who do not give reference to Shtrikman \& Thomas (1965a,b). Grimmer (1993) extended their approach to thermoelectric transport properties. Consider a crystal in which an electric current with density $\mathbf{j}$ flows and a temperature gradient grad $T$ exists. These produce a heat current with density $\mathbf{h}$ and a gradient of the electrochemical potential $\bar{\varphi}=\varphi-\mu / e$, where $\varphi$ is the electric potential, $\mu$ is the chemical potential and $-e$ is the charge of the electron. Assuming linear relationships and using Cartesian coordinates we can write [equation (4) in Grimmer (1993)]

$$
\begin{gathered}
-\frac{\partial \bar{\varphi}}{\partial x_{i}}=\rho_{i k}(\mathbf{H}, \mathbf{M}) j_{k}+\Sigma_{i k}^{\prime}(\mathbf{H}, \mathbf{M}) \frac{\partial T}{\partial x_{k}}, \\
h_{i}=T \Sigma_{i k}^{\prime \prime}(\mathbf{H}, \mathbf{M}) j_{k}-k_{i k}(\mathbf{H}, \mathbf{M}) \frac{\partial T}{\partial x_{k}}
\end{gathered}
$$

where the property tensors depend on the external magnetic field $\mathbf{H}$, assumed to be constant or varying slowly as a function of space and time, and on the time-averaged magnetization field $\mathbf{M}$ describing the magnetic configuration of the crystal. Time inversion (time reversal) $1^{\prime}$ relates crystals with magnetic moments of opposite sign; $\mathbf{M}$ and $\mathbf{H}$ change sign under $1^{\prime}$. Whereas $\mathbf{M}$ varies over a (primitive) magnetic unit cell for magnetically ordered crystals and is invariant under space inversion $\overline{1}$ only for centrosymmetric crystals, the vector $\mathbf{H}$ is approximately constant in a magnetic unit cell; $\mathbf{H}$ is a so-called 
magnetic vector, i.e. it changes sign under $1^{\prime}$ and is invariant under space inversion $\overline{1}$.

We introduce a $6 \times 6$ matrix $R_{\mu \nu}$ by defining

$$
R_{i k}=\rho_{i k}, R_{i k+3}=\Sigma_{i k}^{\prime}, R_{i+3 k}=\Sigma_{i k}^{\prime \prime}, R_{i+3 k+3}=k_{i k},
$$

where $i, k=1,2,3$. Defining

$$
R_{\mu \nu}^{ \pm}(\mathbf{H}, \mathbf{M})=\frac{1}{2}\left[R_{\mu \nu}(\mathbf{H}, \mathbf{M}) \pm R_{\mu \nu}(-\mathbf{H},-\mathbf{M})\right],
$$

we split the matrix $R_{\mu \nu}$ into a part $R_{\mu \nu}^{+}$that is invariant and a part $R_{\mu \nu}^{-}$that changes sign under time reversal $1^{\prime}$. The Onsager relations can then be written as (Shtrikman \& Thomas, $1965 a, b)$

$$
R_{\mu \nu}(\mathbf{H}, \mathbf{M})=R_{v \mu}(-\mathbf{H},-\mathbf{M}) .
$$

It follows that $R_{\mu \nu}^{ \pm}$can also be written as

$$
R_{\mu \nu}^{ \pm}(\mathbf{H}, \mathbf{M})=\frac{1}{2}\left[R_{\mu \nu}(\mathbf{H}, \mathbf{M}) \pm R_{v \mu}(\mathbf{H}, \mathbf{M})\right]
$$

i.e. $R_{\mu \nu}^{+}$is symmetric and $R_{\mu \nu}^{-}$antisymmetric in $\mu$ and $\nu$.

For small magnetic fields the matrices $R_{\mu \nu}^{ \pm}$can be expanded in powers of $\mathbf{H}$,

$$
\begin{aligned}
R_{\mu \nu}^{ \pm}\left(\mathbf{H}, \mathbf{M}_{0}\right)= & R_{\mu \nu}^{0 \pm}\left(\mathbf{M}_{0}\right)+R_{\mu \nu l}^{ \pm}\left(\mathbf{M}_{0}\right) H_{l}+R_{\mu \nu l m}^{ \pm}\left(\mathbf{M}_{0}\right) H_{l} H_{m} \\
& +R_{\mu \nu l m n}^{ \pm}\left(\mathbf{M}_{0}\right) H_{l} H_{m} H_{n}+\ldots,
\end{aligned}
$$

where $\mathbf{M}_{0}$ is the magnetization of the crystal for $\mathbf{H}=0$. Note that $\mathbf{M}_{0}=0$ for dia- and paramagnetic crystals. It follows from equation (3) that

$$
\begin{aligned}
& R_{\mu \nu}^{0 \pm}\left(\mathbf{M}_{0}\right)=\frac{1}{2}\left[R_{\mu \nu}^{0}\left(\mathbf{M}_{0}\right) \pm R_{\mu \nu}^{0}\left(-\mathbf{M}_{0}\right)\right]= \pm R_{\mu \nu}^{0 \pm}\left(-\mathbf{M}_{0}\right), \\
& R_{\mu \nu l}^{ \pm}\left(\mathbf{M}_{0}\right)=\frac{1}{2}\left[R_{\mu \nu l}\left(\mathbf{M}_{0}\right) \mp R_{\mu \nu l}\left(-\mathbf{M}_{0}\right)\right]=\mp R_{\mu \nu l}^{ \pm}\left(-\mathbf{M}_{0}\right) .
\end{aligned}
$$

Relations similar to equation (7a) hold for all terms connected with even powers of $\mathbf{H}$, relations similar to equation $(7 b)$ for all terms connected with odd powers of $\mathbf{H}$. Because $\mathbf{H}$ changes sign under time reversal $1^{\prime}$ it follows that $R_{\mu \nu}^{0+}$ and $R_{\mu \nu l}^{-}$are invariant under $1^{\prime}$. More generally, $R_{\mu \nu l_{1} \ldots l_{n}}^{+}$is invariant under $1^{\prime}$ if $n$ is even, $R_{\mu \nu l_{1} \ldots l_{n}}^{-}$is invariant under $1^{\prime}$ if $n$ is odd.

Consider first the electric transport properties of a crystal described by $\rho_{i k}(\mathbf{H}, \mathbf{M}),(i, k=1,2,3)$, i.e. by $R_{i k}(\mathbf{H}, \mathbf{M})$ according to equation (2). According to equation (1a) the tensor $\rho_{i k}(\mathbf{H}, \mathbf{M}) \operatorname{gives} \operatorname{grad} \bar{\varphi}$ in a crystal in which an electric current $\mathbf{j}$ flows and in which the temperature is constant throughout the crystal. For $\mu=i$ and $\nu=k$ we can replace $R$ by $\rho$ in equations (3)-(7a), (7b). The part invariant under $1^{\prime}$, $\rho_{i k}^{+}(\mathbf{H}, \mathbf{M})$, is symmetric, the part changing sign, $\rho_{i k}^{-}(\mathbf{H}, \mathbf{M})$, is antisymmetric in $i$ and $k$.

As shown in Shtrikman \& Thomas $(1965 a, b)$, only the symmetric part $\rho_{i k}^{+}(\mathbf{H}, \mathbf{M})$ contributes to energy dissipation,

$$
Q=-j_{i} \frac{\partial \bar{\varphi}}{\partial x_{i}}=j_{i} \rho_{i k} j_{k}=j_{i} \rho_{k i} j_{k}=j_{i} \rho_{i k}^{+} j_{k} .
$$

The symmetric part $\rho_{i k}^{+}(\mathbf{H}, \mathbf{M})$ describes the electric resistance, the antisymmetric part $\rho_{i k}^{-}(\mathbf{H}, \mathbf{M})$ the Hall effect, and their dependence on $\mathbf{H}$. If $\mathbf{M}=0$, it follows from equation (6) that $\rho^{+}$contains only terms connected with even powers and $\rho^{-}$ only terms connected with odd powers of $\mathbf{H}$. For crystals with $\mathbf{M}=0$ it follows that magnetoresistance linear in $\mathbf{H}$ is not possible and that the Hall effect must vanish identically if $\mathbf{H}=0$.

Equations $(7 a),(7 b)$ show that $\rho_{i k}^{0+}, \rho_{i k l}^{-}$and $\rho_{i k l m}^{+}$are invariant under time reversal $1^{\prime}$ whereas $\rho_{i k}^{0-}, \rho_{i k l}^{+}$and $\rho_{i k l m}^{-}$ change sign. The tensors symmetric in $i$ and $k$ describe electric resistance $\left[\rho_{i k}^{0+}\left(\mathbf{M}_{0}\right)\right]$, linear magnetoresistance $\left[\rho_{i k l}^{+}\left(\mathbf{M}_{0}\right)\right]$ and (quadratic) magnetoresistance $\left[\rho_{i k l m}^{+}\left(\mathbf{M}_{0}\right)\right]$, the tensors antisymmetric in $i$ and $k$ describe the spontaneous Hall effect $\left[\rho_{i k}^{0-}\left(\mathbf{M}_{0}\right)\right]$, (ordinary) Hall effect $\left[\rho_{i k l}^{-}\left(\mathbf{M}_{0}\right)\right]$ and (quadratic) anomalous Hall effect $\left[\rho_{i k l m}^{-}\left(\mathbf{M}_{0}\right)\right]$.

Similarly, the thermal transport properties are described by $k_{i j}(\mathbf{H}, \mathbf{M}),(i, j=1,2,3)$, i.e. by $R_{i+3 j+3}(\mathbf{H}, \mathbf{M})$ according to equation (2). For $\mu=i+3$ and $v=j+3$ we can replace $R$ by $k$ in equations (3)-(7a), (7b). The part invariant under $1^{\prime}$, $k_{i j}^{+}(\mathbf{H}, \mathbf{M})$, is symmetric, the part changing sign, $k_{i j}^{-}(\mathbf{H}, \mathbf{M})$, is antisymmetric in $i$ and $j$. Equations (7a), (7b) show that $k_{i j}^{0+}, k_{i j l}^{-}$and $k_{i j l m}^{+}$are invariant under time reversal $1^{\prime}$ whereas $k_{i j}^{0-}, k_{i j l}^{+}$and $k_{i j m}^{-}$change sign. The tensors symmetric in $i$ and $j$ describe heat conductivity $\left[k_{i j}^{0+}\left(\mathbf{M}_{0}\right)\right]$, linear magnetoheat-conductivity $\left[k_{i j l}^{+}\left(\mathbf{M}_{0}\right)\right]$ and (quadratic) magneto-heatconductivity $\left[\rho_{i k l m}^{+}\left(\mathbf{M}_{0}\right)\right]$, the tensors antisymmetric in $i$ and $j$ describe the spontaneous Righi-Leduc effect $\left[k_{i j}^{0-}\left(\mathbf{M}_{0}\right)\right]$, (ordinary) Righi-Leduc effect $\left[k_{i j l}^{-}\left(\mathbf{M}_{0}\right)\right]$ and (quadratic) anomalous Righi-Leduc effect $\left[k_{i k l m}^{-}\left(\mathbf{M}_{0}\right)\right]$.

The thermoelectric transport properties are described by $\Sigma_{i j}^{\prime \prime}(\mathbf{H}, \mathbf{M}),(i, j=1,2,3)$, i.e. by $R_{i+3 j}(\mathbf{H}, \mathbf{M})$ according to equation (2) and by $\Sigma_{j i}^{\prime}(\mathbf{H}, \mathbf{M}),(i, j=1,2,3)$, i.e. by $R_{j i+3}(\mathbf{H}, \mathbf{M})$. For $\mu=i+3$ and $v=j$ we can replace $R$ by $\Sigma^{\prime \prime}$ in equations (3)-(7a), (7b); for $\mu=j$ and $v=i+3$ we can replace $R$ by $\Sigma^{\prime}$ in equations (3)-(7a), (7b). The part invariant under $1^{\prime}$ satisfies $\Sigma_{i j}^{\prime \prime+}(\mathbf{H}, \mathbf{M})=\Sigma_{j i}^{\prime+}(\mathbf{H}, \mathbf{M})$, the part changing sign satisfies $\Sigma_{i j}^{\prime \prime}(\mathbf{H}, \mathbf{M})=-\Sigma_{j i}^{\prime-}(\mathbf{H}, \mathbf{M})$. Equations $(7 a),(7 b)$ show that $\Sigma_{i j}^{\prime 0+}, \Sigma_{i j}^{\prime \prime 0+}, \Sigma_{i j l}^{\prime-}, \Sigma_{i j l}^{\prime \prime-}, \Sigma_{i j l m}^{\prime+}$ and $\Sigma_{i j l m}^{\prime \prime+}$ are invariant under time reversal $1^{\prime}$ whereas $\Sigma_{i j}^{\prime 0-}, \Sigma_{i j}^{\prime \prime 0-}, \Sigma_{i j l}^{\prime+}, \Sigma_{i j l}^{\prime \prime+}, \Sigma_{i j m}^{\prime-}$ and $\Sigma_{i j l m}^{\prime \prime}$ change sign. Note that these tensors have no internal symmetry. The effects described are the Seebeck effect $\left(\Sigma_{i j}^{0+}\right)$, Peltier effect $\left(T \Sigma_{i j}^{\prime \prime 0+}\right)$, linear magneto-Seebeck effect $\left(\Sigma_{i j l}^{\prime+}\right)$, linear magneto-Peltier effect $\left(T \Sigma_{i j l}^{\prime \prime+}\right)$, (quadratic) magnetoSeebeck effect $\left(\Sigma_{i j l m}^{\prime+}\right)$, (quadratic) magneto-Peltier effect $\left(T \Sigma_{i j l m}^{\prime \prime+}\right)$, spontaneous Nernst effect $\left(\Sigma_{i j}^{\prime 0-}\right)$, spontaneous Ettingshausen effect $\left(T \Sigma_{i j}^{\prime \prime 0-}\right)$, (ordinary) Nernst effect $\left(\Sigma_{i j l}^{\prime-}\right)$, (ordinary) Ettingshausen effect $\left(T \Sigma_{i j l}^{\prime \prime-}\right)$, (quadratic) anomalous Nernst effect $\left(\Sigma_{i j m}^{\prime-}\right)$ and (quadratic) anomalous Ettingshausen effect $\left(T \sum_{i j l m}^{\prime \prime \prime}\right)$.

The above-mentioned 12 effects invariant under time inversion $1^{\prime}$ are also invariant under space inversion $\overline{1}$ (and, therefore, also under space-time inversion $\overline{1}^{\prime}$ ). The tensors describing such effects are called even (Sirotin \& Shaskolskaya, 1982); they can occur in crystals with any of the 122 space-time point groups. The 12 effects changing sign under time inversion $1^{\prime}$ are invariant under space inversion $\overline{1}$ (and, therefore, change sign under space-time inversion $\overline{1}^{\prime}$ ). The tensors describing such effects are called magnetic (Sirotin \& Shaskolskaya, 1982); they cannot occur in crystals with any of the 53 space-time point groups containing $1^{\prime}$ or $\overline{1}^{\prime}$ as a separate element. Magnetically ordered crystals have one of the 90 magnetic point groups (MPGs) that do not contain time 
Table 1

The tensors describing thermoelectric transport properties considered in $\S 3$.

\begin{tabular}{|c|c|c|c|c|}
\hline \multicolumn{2}{|c|}{$\begin{array}{l}\text { Even tensors (invariant under } \overline{1} \text { and } 1^{\prime} \text { ) } \\
\text { describing effects allowed in all } 122 \text { point groups }\end{array}$} & \multicolumn{2}{|c|}{$\begin{array}{l}\text { Magnetic tensors (invariant under } \overline{1} \text {, } \\
\text { change sign under } 1^{\prime} \text { ) describing }\end{array}$} & \multirow{2}{*}{$\begin{array}{l}\text { Number of point groups } \\
\text { allowing the effect }\end{array}$} \\
\hline$\rho_{i j}^{0+}$ & Electric resistance $(\mathbf{H}=0)$ & $\rho_{i j}^{0-}$ & Spontaneous Hall effect $(\mathbf{H}=0)$ & \\
\hline$\rho_{i j l}^{-}$ & (Ordinary) Hall effect & $\rho_{i j l}^{+}$ & Linear magnetoresistance & 66 \\
\hline$\rho_{i j l m}^{+}$ & (Quadratic) magnetoresistance & $\rho_{\overline{i j l m}}^{-}$ & (Quadratic) anomalous Hall effect & 66 \\
\hline$k_{i j}^{0+}$ & Heat conductivity $(\mathbf{H}=0)$ & $k_{i j}^{0-}$ & Spontaneous Righi-Leduc effect $(\mathbf{H}=0)$ & 31 \\
\hline$k_{i j l}^{-}$ & (Ordinary) Righi-Leduc effect & $k_{i j l}^{+}$ & Linear magneto-heat-conductivity & 66 \\
\hline$\Sigma_{i j}^{\prime 0+}$ & Seebeck effect $(\mathbf{H}=0)$ & $\Sigma_{i j}^{\prime 0-}$ & Spontaneous Nernst effect $(\mathbf{H}=0)$ & 58 \\
\hline$\Sigma_{i j l}^{\prime-}$ & (Ordinary) Nernst effect & $\Sigma_{i j l}^{\prime+}$ & Linear magneto-Seebeck effect & 69 \\
\hline$\Sigma_{i j l m}^{\prime+}$ & (Quadratic) magneto-Seebeck effect & $\Sigma_{i j l m}^{\prime-}$ & (Quadratic) anomalous Nernst effect & 69 \\
\hline$T \Sigma_{i j}^{\prime \prime 0+}$ & Peltier effect $(\mathbf{H}=0)$ & $T \Sigma_{i j}^{\prime \prime 0-}$ & Spontaneous Ettingshausen effect $(\mathbf{H}=0)$ & 58 \\
\hline$T \Sigma_{i j l}^{\prime \prime-}$ & (Ordinary) Ettingshausen effect & $T \Sigma_{i j l}^{\prime \prime+}$ & Linear magneto-Peltier effect & 69 \\
\hline
\end{tabular}

inversion $1^{\prime}$ as a separate element. If $\mathbf{H}=0$, spontaneous Hall and Righi-Leduc effects are possible for the 31 MPGs allowing ferromagnetism, spontaneous Nernst and Ettingshausen effects are allowed for 58 MPGs. Whereas magneto-resistance, magneto-heat-conductivity, magnetoSeebeck and magneto-Peltier effect are of even order in $\mathbf{H}$ in crystals without magnetic order, such effects linear in $\mathbf{H}$ are possible in the case of magnetoresistance and magneto-heat-conductivity for the 66 MPGs allowing piezomagnetism, and in the case of the magneto-Seebeck and magneto-Peltier effects for all $122-53=69$ MPGs that do not contain space-time inversion $\overline{1}^{\prime}$ as a separate element. Also the (quadratic) anomalous Hall and Righi-Leduc effects occur in the 66 MPGs allowing piezomagnetism, whereas the (quadratic) anomalous Nernst and Ettingshausen effects occur for all 69 MPGs that do not contain space-time inversion $\overline{1}^{\prime}$ as a separate element. These effects, which occur only in magnetically ordered crystals belonging to certain MPGs, are described by magnetic tensors. They are summarized in Table 1.

Kleiner (1966), Malinowski (1986), Seemann et al. (2015) and the Bilbao Crystallographic Server (2017) consider the restrictions on the sum of the even and the magnetic tensor given in the same row of Table 1 . It will be shown that the two tensors can be determined separately. The restrictions that follow from the Neumann principle [see e.g. Nye (1985) or section 3.2.2.1 in International Tables for Crystallography (2016)] for the form of the tensors describing the effects occurring already at $\mathbf{H}=0$ or linear in $\mathbf{H}$ have been derived by Grimmer (1993), where the results are given in Table 1 in a very compact form using graphic symbols that allow one to see immediately the components with equal or opposite values and the number of independent components. These results will be presented here in a more user-friendly form, for additional orientations of the Cartesian coordinate system, and augmented by the restrictions for the tensors describing the effects quadratic in $\mathbf{H}$.

\section{Symmetry restrictions on tensors describing crystal properties}

This section discusses basic facts and conventions important for the unambiguous interpretation of the restrictions that follow from the Neumann principle on the form of property tensors for different choices of the Cartesian coordinate system.

Neglecting magnetic order, crystals can be classified according to their symmetry into 230 'crystallographic space groups' ['Fedorov groups' in Russian literature, see e.g. Vainshtein (1996)]; neglecting also translational symmetry they fall into 32 crystal classes corresponding to the 32 crystallographic point groups. Two kinds of tensors can be distinguished according to whether they are invariant or change sign under space inversion $\overline{1}$. They have been referred to as (plus or) $p$ tensors and (minus or) $m$ tensors (Grimmer, 1991) or as even and odd tensors (Sirotin \& Shaskolskaya, 1982). Adding $\overline{1}$ to the generators of a point group, one of the 11 centrosymmetric point groups is obtained. Point groups associated in this way to the same centrosymmetric point group are said to belong to the same Laue class. Each Laue class contains exactly one point group containing only rotations; it is convenient to use its symbol to denote the Laue class.

Whereas the restrictions imposed by the Neumann principle on tensors describing crystal properties depend for even tensors of given rank and internal symmetry only on the Laue class to which the point group belongs, odd tensors vanish for the 11 centrosymmetric crystal classes and satisfy generally different restrictions for the remaining 21 classes. Odd tensors of low, non-zero rank vanish also for some of the 21 noncentrosymmetric crystal classes, e.g. polar vectors (i.e. odd tensors of rank 1) describing crystal properties can exist only for the ten polar point groups 1, 2, 3, 4, 6, m, mm2, 3m, 4mm and $6 \mathrm{~mm}$. [See Table 3.2.2.1 in International Tables for Crystallography (2016)]. Crystals belonging to one of these ten 
Table 2

The four kinds of tensors defined by their behaviour under inversions.

Invariance of the tensor is denoted by + , sign change by - .

\begin{tabular}{|c|c|c|c|c|}
\hline $\begin{array}{l}\text { Space } \\
\text { inversion } \overline{1}\end{array}$ & $\begin{array}{l}\text { Time } \\
\text { inversion } 1^{\prime}\end{array}$ & $\begin{array}{l}\text { Space-time } \\
\text { inversion } \overline{1}^{\prime}\end{array}$ & Name used by Grimmer (1991) & $\begin{array}{l}\text { Name used by } \\
\text { Sirotin \& Shaskolskaya (1982) }\end{array}$ \\
\hline+ & + & + & $i$ tensor, invariant under all inversions & Even tensor \\
\hline+ & - & - & $s$ tensor, invariant under space inversion & Magnetic tensor \\
\hline- & + & - & $t$ tensor, invariant under time inversion & Electric tensor \\
\hline- & - & + & $u$ tensor, invariant under space-time inversion & Magnetoelectric tensor \\
\hline
\end{tabular}

crystal classes are pyro-, ferro- or ferri-electric (Schmid, 1973).

To formulate the restrictions imposed by the Neumann principle, a Cartesian coordinate system (CCS) is introduced, the axes of which are given by the international (HermannMauguin) symbol of the point group. An entry in the Hermann-Mauguin (HM) symbol consists (apart from possible primes and bars) of a number $N=1,2,3,4$ or 6 or the letter $m$ or $N / m$. The usual conventions will be followed: in the monoclinic and orthorhombic crystal systems, the $x, y$ and $z$ axes of the CCS are parallel to the symmetry axes given in the first, second and third entries, respectively. In the monoclinic system, there is only one symmetry axis, which is usually chosen as $y$, and a short HM symbol with only one entry is most often used, e.g. $2 / m$ instead of $12 / m 1$. In the trigonal and hexagonal systems, the $z, x$ and $y$ axes are parallel to the symmetry axes given in the first, second and third entries, respectively. In the tetragonal system, the $z$ axis is parallel to the symmetry axis given in the first entry; the $x$ and $y$ axes are parallel to the symmetry axes given in the second entry, which appear in two mutually perpendicular directions. In the cubic system, the symmetry axes given in the first entry appear in three mutually perpendicular directions; the $x, y$ and $z$ axes of the CCS are chosen parallel to these directions (Nye, 1985; Grimmer, 1991; Borovik-Romanov et al., 2014). For certain point groups, different HM symbols are possible, e.g. $\overline{6} m 2$ or $\overline{6} 2 \mathrm{~m}$, corresponding to inequivalent choices of the CCS. Only the standard symbol $\overline{6} \mathrm{~m} 2$ is used in Nye (1985) and it is stated explicitly if a CCS is used that deviates from the conventions given above. Confusion may arise from the conventions proposed in the IEEE Standard on Piezoelectricity (1988), where the standard HM symbol $\overline{6} m 2$ is used together with a CCS corresponding to $\overline{6} 2 m$ according to the conventions given above.

Grimmer (1991) has shown how $9 \times 3=27$ different forms of an odd property tensor of given rank and internal symmetry can be derived from $6 \times 2=12$ forms for the corresponding even property tensor. This is illustrated in his Fig. 1 for tensors of third rank, symmetric in two of its three indices (Jahn symbol $V\left[V^{2}\right]$, see Jahn, 1949) and in his Fig. 2 for the fourthrank tensor $\left[V^{2}\right]^{2}$. Similarly, the forms of $\left[V^{2}\right],\left\{V^{2}\right\}, V^{2}, V\left[V^{2}\right]$, $V\left\{V^{2}\right\}$ and $V^{3}$ are given in Table 1 of Grimmer (1993). Even and odd property tensors of given rank and internal symmetry satisfy the same restrictions for pure rotation groups; these are given for standard orientations, where the monoclinic group 2 appears in two forms 121 and 112 , leading to $11+1=12$ forms.
Also the forms of odd tensors for some of the other ten noncentrosymmetric point groups are given for several orientations: $1 m 1$ and $11 m ; m m 2, m 2 m$ and $2 m m ; \overline{4} 2 m$ and $\overline{4} m 2 ; \overline{6} 2 m$ and $\overline{6} \mathrm{~m} 2$, leading to a total of $12+15=27$ forms for odd tensors. Note that the forms given in Grimmer $(1991,1993)$ for 32 and $3 m$ hold for 321 and $3 m 1$, respectively. In the present paper, also the forms for 312 and $31 \mathrm{~m}$ will be given.

Crystals without magnetic order (dia- and paramagnetic crystals) are invariant under the inversion of magnetic moments, usually referred to as time inversion $1^{\prime}$ (Grimmer, 1993). Such crystals belong to one of the 230 space-time groups obtained from the ordinary space groups by adding $1^{\prime}$ as additional generator. Interpreting $1^{\prime}$ for graphical representations as exchanging black and white, the 230 space groups containing the element 1 ' are called 'grey' (category I), and the 230 ordinary space groups 'monochrome' (category II). In addition, there are 1191 'black-white' space groups containing $1^{\prime}$ only in combination with other operations, in total 1651 'magnetic space groups' (see Kleiner, 1966, or Borovik-Romanov et al., 2014), referred to as 'Shubnikov groups' in Russian literature (see e.g. Vainshtein, 1996). Among the 1191 black-white space groups 517 contain 1' in combination with translations (category III $^{b}$ ), the other 674 do not contain $1^{\prime}$ combined with translations (category $\mathrm{III}^{a}$ ). According to Kleiner (1966), category $\mathrm{III}^{a}$ can be further split into III $^{a 1}$ consisting of the 422 groups that do not contain $\overline{1}^{\prime}$ and $\mathrm{III}^{a 2}$ consisting of the 252 groups containing $\overline{1}^{\prime}$.

Neglecting translational symmetry, the 1651 magnetic space groups fall into 122 crystal classes corresponding to the 122 space-time point groups, 32 of which are grey, 32 are monochrome and 58 black-white. If the space group is grey the point group is also grey, if the space group is monochrome the point group is also monochrome; space groups of category $\mathrm{III}^{a}$ have black-white point groups whereas space groups of category $\mathrm{III}^{b}$ have grey point groups [see also Fig. 1 of Kleiner (1966)].

Four kinds of tensors can be distinguished according to whether they are invariant or change sign under space inversion $\overline{1}$, time inversion $1^{\prime}$ and space-time inversion $\overline{1}^{\prime}$, as shown in Table 2 (see also Table 3 in Grimmer, 1991). Note that $i$ and $s$ tensors are 'even', $t$ and $u$ tensors 'odd' as far as their behaviour under $\overline{1}$ is concerned. In the following, we shall use the term 'even tensor' in the sense of Sirotin \& Shaskolskaya (1982).

Considering that the 11 Laue classes were originally defined as determining the symmetry of X-ray photographs if Friedel's 
Table 3

The 69 space-time point groups for which magnetic tensors can exist and their subdivision into 21 magnetic form classes and two categories.

The point groups in the first category are monochrome; they correspond to space-group category II. The point groups in the second category are blackwhite and do not contain $\overline{1}^{\prime}$; they correspond to space-group category III ${ }^{a 1}$. The third column begins with the point group containing only rotations (possibly combined with time inversion) and ends with the centrosymmetric point group. The magnetic form classes and the corresponding point groups are listed not only for the standard orientation of the Cartesian coordinate system but, in parentheses, also for the other orientations that will be considered.

\begin{tabular}{|c|c|c|}
\hline Category & Magnetic form class & Point groups \\
\hline \multirow[t]{14}{*}{ II } & 1 & $1, \overline{1}$ \\
\hline & (211) & $(211),(m 11),(2 / m 11)$ \\
\hline & 121 & $121,1 m 1,12 / m 1$ \\
\hline & $(112)$ & (112), $(11 m),(112 / m)$ \\
\hline & 222 & $222, m m 2,(2 m m),(m 2 m), m m m$ \\
\hline & 3 & $3, \overline{3}$ \\
\hline & 321 & $321,3 m 1, \overline{3} m 1$ \\
\hline & (312) & $(312),(31 m),(\overline{3} 1 m)$ \\
\hline & 4 & $4, \overline{4}, 4 / m$ \\
\hline & 422 & $422,4 m m, \overline{4} 2 m,(\overline{4} \mathrm{~m} 2), 4 / \mathrm{mmm}$ \\
\hline & 6 & $6, \overline{6}, 6 / m$ \\
\hline & 622 & $622,6 \mathrm{~mm}, \overline{6} \mathrm{~m} 2,(\overline{6} 2 \mathrm{~m}), 6 / \mathrm{mmm}$ \\
\hline & 23 & $23, m \overline{3}$ \\
\hline & 432 & $432, \overline{4} 3 m, m \overline{3} m$ \\
\hline \multirow[t]{17}{*}{$\mathrm{III}^{a 1}$} & $\left(2^{\prime} 11\right)$ & $\left(2^{\prime} 11\right),\left(m^{\prime} 11\right),\left(2^{\prime} / m^{\prime} 11\right)$ \\
\hline & $12^{\prime} 1$ & $12^{\prime} 1,1 m^{\prime} 1,12^{\prime} / m^{\prime} 1$ \\
\hline & $\left(112^{\prime}\right)$ & $\left(112^{\prime}\right),\left(11 m^{\prime}\right),\left(112^{\prime} / m^{\prime}\right)$ \\
\hline & $\left(22^{\prime} 2^{\prime}\right)$ & $\left(22^{\prime} 2^{\prime}\right),\left(2 m^{\prime} m^{\prime}\right),\left(m m^{\prime} 2^{\prime}\right),\left(m 2^{\prime} m^{\prime}\right),\left(m m^{\prime} m^{\prime}\right)$ \\
\hline & $\left(2^{\prime} 22^{\prime}\right)$ & $\left(2^{\prime} 22^{\prime}\right),\left(m^{\prime} 2 m^{\prime}\right),\left(2^{\prime} m m^{\prime}\right),\left(m^{\prime} m 2^{\prime}\right),\left(m^{\prime} m m^{\prime}\right)$ \\
\hline & $2^{\prime} 2^{\prime} 2$ & $2^{\prime} 2^{\prime} 2, m^{\prime} m^{\prime} 2, m^{\prime} 2^{\prime} m,\left(2^{\prime} m^{\prime} m\right), m^{\prime} m^{\prime} m$ \\
\hline & $4^{\prime}$ & $4^{\prime}, \overline{4}^{\prime}, 4^{\prime} / m$ \\
\hline & $4^{\prime} 22^{\prime}$ & $4^{\prime} 22^{\prime}, 4^{\prime} m m^{\prime}, \overline{4}^{\prime} 2 m^{\prime}, \overline{4}^{\prime} m 2^{\prime}, 4^{\prime} / \mathrm{mmm}^{\prime}$ \\
\hline & $\left(4^{\prime} 2^{\prime} 2\right)$ & $\left(4^{\prime} 2^{\prime} 2\right),\left(4^{\prime} m^{\prime} m\right),\left(\overline{4}^{\prime} m^{\prime} 2\right),\left(\overline{4}^{\prime} 2^{\prime} m\right),\left(4^{\prime} / m m^{\prime} m\right)$ \\
\hline & $32^{\prime} 1$ & $32^{\prime} 1,3 m^{\prime} 1, \overline{3} m^{\prime} 1$ \\
\hline & $\left(312^{\prime}\right)$ & $\left(312^{\prime}\right),\left(31 m^{\prime}\right),\left(\overline{3} 1 m^{\prime}\right)$ \\
\hline & $42^{\prime} 2^{\prime}$ & $42^{\prime} 2^{\prime}, 4 m^{\prime} m^{\prime}, \overline{4} 2^{\prime} m^{\prime},\left(\overline{4} m^{\prime} 2^{\prime}\right), 4 / m m^{\prime} m^{\prime}$ \\
\hline & $62^{\prime} 2^{\prime}$ & $62^{\prime} 2^{\prime}, 6 m^{\prime} m^{\prime}, \overline{6} m^{\prime} 2^{\prime},\left(\overline{6} 2^{\prime} m^{\prime}\right), 6 / m m^{\prime} m^{\prime}$ \\
\hline & $6^{\prime}$ & $6^{\prime}, \overline{6}^{\prime}, 6^{\prime} / m^{\prime}$ \\
\hline & $6^{\prime} 22^{\prime}$ & $6^{\prime} 22^{\prime}, 6^{\prime} m m^{\prime}, \overline{6}^{\prime} m 2^{\prime}, \overline{6}^{\prime} 2 m^{\prime}, 6^{\prime} / m^{\prime} m m^{\prime}$ \\
\hline & $\left(6^{\prime} 2^{\prime} 2\right)$ & $\left(6^{\prime} 2^{\prime} 2\right),\left(6^{\prime} m^{\prime} m\right),\left(\overline{6}^{\prime} 2^{\prime} m\right),\left(\overline{6}^{\prime} m^{\prime} 2\right),\left(6^{\prime} / m^{\prime} m^{\prime} m\right)$ \\
\hline & $4^{\prime} 32^{\prime}$ & $4^{\prime} 32^{\prime}, \overline{4}^{\prime} 3 m^{\prime}, m \overline{3} m^{\prime}$ \\
\hline
\end{tabular}

law is valid, it is natural to extend their definition as follows to the 122 space-time point groups: adding $\overline{1}$ and $1^{\prime}$ to the generators of a point group, one of the 11 point groups containing $\overline{1}, 1^{\prime}$ and $\overline{1}^{\prime}$ is obtained. Point groups associated in this way to the same grey, centrosymmetric point group are said to belong to the same (space-time) Laue class. Each of these 11 Laue classes contains exactly one group consisting of rotations only. We denote each Laue class by adding $1^{\prime}$ to the HM symbol of its pure rotation.
With this definition of (space-time) Laue class it remains true that the restrictions imposed by the Neumann principle on tensors describing crystal properties depend for even tensors of given rank and internal symmetry only on the Laue class to which the point group belongs. Magnetic $(s)$ tensors vanish for the 53 point groups that contain $1^{\prime}$ and/or $\overline{1}^{\prime}$, electric $(t)$ tensors vanish for the 53 point groups that contain $\overline{1}$ and/or $\overline{1}^{\prime}$, magnetoelectric $(u)$ tensors vanish for the 53 point groups that contain $\overline{1}$ and/or $1^{\prime}$. In each of these three cases there remain 69 point groups, which may be grouped into 21 form classes according to the restrictions imposed by the Neumann principle. For magnetic tensors this grouping has been given explicitly in Table II of Kleiner (1966) and in Table 1 of Grimmer (1993) and for all three cases in Fig. 1 of Grimmer (1994).

11 of the 21 magnetic form classes contain only monochrome point groups. Each of these 11 classes contains exactly one group consisting of rotations only; it is convenient to use its symbol to denote the magnetic form class. (Note that these 11 magnetic form classes look like the Laue classes of ordinary point groups. However, only magnetically ordered structures that have no symmetry operations involving $1^{\prime}$ belong to one of these 11 magnetic form classes.) The other ten magnetic form classes consist of black-white point groups. Each of these ten classes contains exactly one group consisting only of rotations and rotations combined with $1^{\prime}$; it is convenient to use its symbol to denote the magnetic form class. We list in Table 3 the 21 magnetic form classes and the 69 corresponding point groups not only for the standard orientation of the CCS but, in parentheses, also for the other orientations that will be considered in this paper. For each of the 69 point groups one HM symbol is considered as standard, other HM symbols designating the same point group appear in parentheses, e.g. $m^{\prime} 2^{\prime} m,\left(2^{\prime} m^{\prime} m\right),\left(m m^{\prime} 2^{\prime}\right),\left(m 2^{\prime} m^{\prime}\right),\left(2^{\prime} m m^{\prime}\right),\left(m^{\prime} m 2^{\prime}\right)$. Note that the restrictions on the form of magnetic property tensors for the 21 magnetic form classes coincide with the restrictions for odd $(m)$ tensors for the 21 non-centrosymmetric crystal classes (Grimmer, 1991).

In the next section thermoelectric transport properties will be considered, distinguishing those described by even tensors from those described by magnetic tensors. The spontaneous Nernst and Ettingshausen effects are allowed in all the magnetic form classes of Table 3 except the last three $\left[6^{\prime}, 6^{\prime} 22^{\prime}\right.$ $\left.\left(6^{\prime} 2^{\prime} 2\right), 4^{\prime} 32^{\prime}\right]$, i.e. in $69-11=58$ point groups; linear magnetoresistance and linear magneto-heat-conductivity are allowed in all magnetic form classes except 432, i.e. in the $69-3=66$ point groups allowing the piezomagnetic effect (see Borovik-Romanov et al., 2014).

The monochrome magnetic form classes $1,2,3,4$ and 6 , which contain 13 point groups, and the black-white magnetic form classes $2^{\prime}, 2^{\prime} 2^{\prime} 2,32^{\prime}, 42^{\prime} 2^{\prime}$ and $62^{\prime} 2^{\prime}$, which contain 18 point groups, allow physical properties described by magnetic vectors. Crystals having one of these $13+18=31$ point groups are pyro-, ferro- or ferrimagnetic (Schmid, 1973) and allow the spontaneous Hall and Righi-Leduc effects. 44 among the 230 space groups of category II have one of those 13 monochrome point groups and 231 among the 422 space groups of category 
III $^{a 1}$ have one of those 18 black-white point groups. The remaining 186 space groups of category II, the remaining 191 space groups of category $\mathrm{III}^{a 1}$, the 252 space groups of category $\mathrm{III}^{a 2}$ and the 517 space groups of category $\mathrm{III}^{b}$ correspond to antiferromagnetic crystals [see section 1.5.2.3 of Borovik-Romanov et al. (2014)]. Crystals with space groups of category I (dia- and paramagnets), category $\mathrm{III}^{b}$ and category $\mathrm{III}^{a 2}$ are not compatible with properties described by magnetic tensors.

\section{Thermoelectric transport properties}

The restrictions that follow from the Neumann principle on the form of the tensors mentioned in Table 1 will be given in this section. The even tensors (listed on the left side of Table 1) will be considered in \$3.1, the magnetic ones (listed on the right side of Table 1) will be considered in $\$ 3.2$.

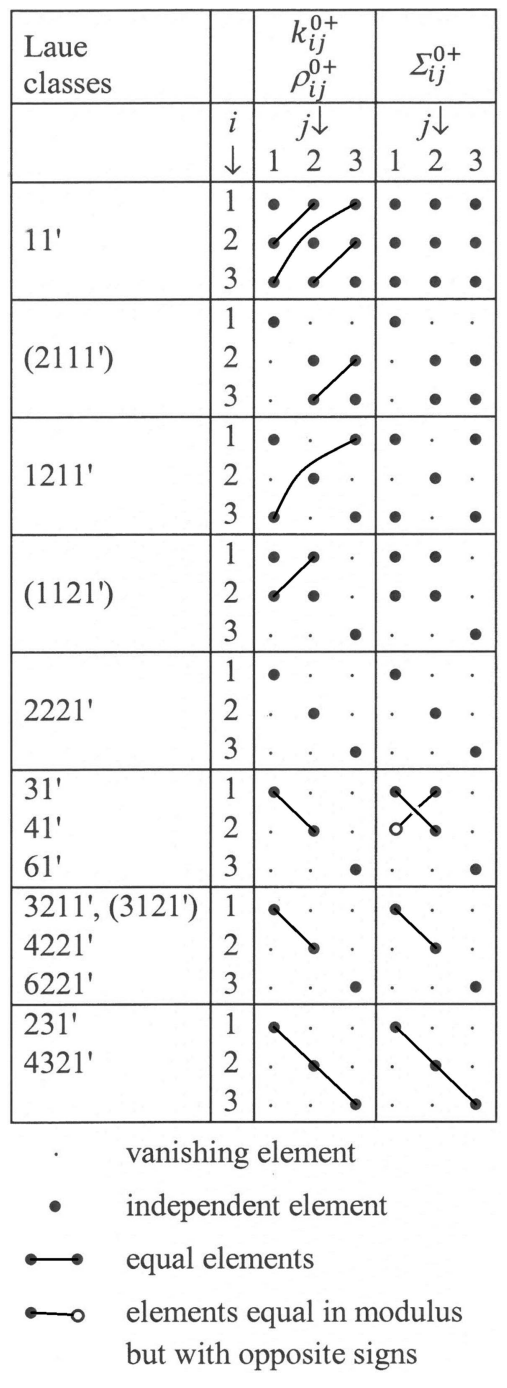

Figure 1

The restrictions satisfied by the property tensors of rank 2 listed on the left-hand side of Table 1 . Laue class symbols between parentheses refer to alternative choices of the Cartesian coordinate system.
3.1. Thermoelectric transport properties described by even tensors

Consider first the effects occurring in the absence of an external magnetic field: electrical resistivity $\rho^{0+}$ and heat conductivity $k^{0+}$ are described by symmetric tensors of rank 2; the Seebeck effect $\Sigma^{\prime 0+}$ and Peltier effect $T \Sigma^{\prime \prime 0+}$ are described by general tensors of rank 2 , where $\Sigma_{i j}^{\prime \prime 0+}=+\Sigma_{j i}^{\prime 0+}$, as follows from equation (5). The form of these tensors is given in Fig. 1.

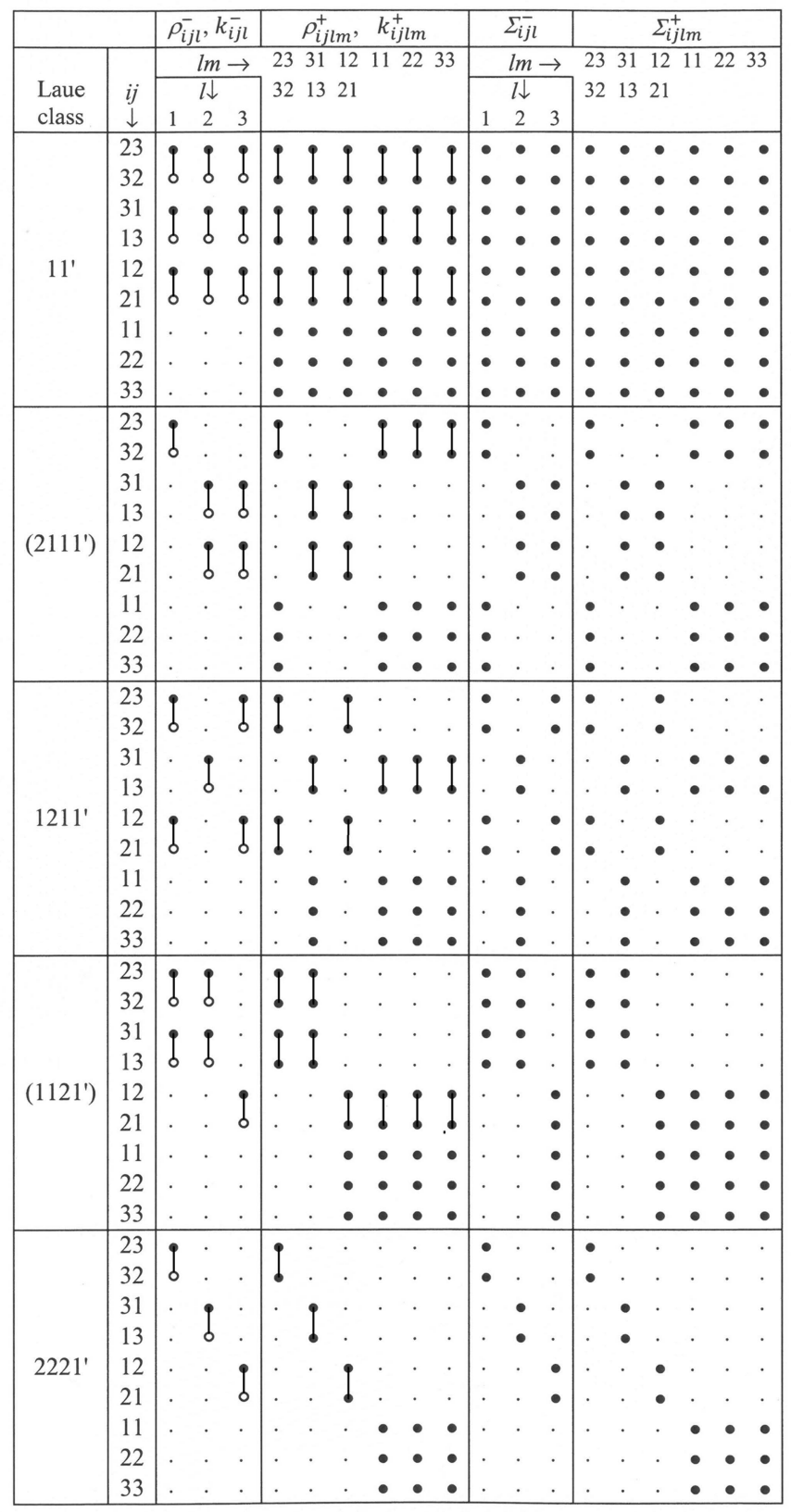

(a)

Figure 2

The restrictions satisfied by the property tensors of ranks 3 and 4 listed on the left-hand side of Table 1. The notation used for the matrix elements is explained at the bottom of Fig. 1. Laue class symbols in parentheses refer to alternative choices of the Cartesian coordinate system. (a) Anorthic, monoclinic and orthorhombic Laue classes. 
In the special cases of cubic crystals (Laue classes 231' and $\left.4321^{\prime}\right)$ the Seebeck tensor $\Sigma^{\prime 0+}$ has only one independent element $\Sigma_{11}^{\prime 0+}=\Sigma_{22}^{\prime 0+}=\Sigma_{33}^{\prime 0+}$ (see Fig. 1). It follows that

$$
\operatorname{grad} \bar{\varphi} \| \operatorname{grad} T \text { if } \mathbf{j}=0 \text {. }
$$

Numerical values for the absolute Seebeck coefficient $S=\Sigma_{11}^{\prime 0+}$ (also called 'absolute thermopower') have been given e.g. in Table XVI of White \& Minges (1997).

Similarly, the Peltier tensor $T \Sigma^{\prime \prime 0+}$ has only one independent element $T \Sigma_{11}^{\prime \prime 0+}=T \Sigma_{22}^{\prime \prime 0+}=T \Sigma_{33}^{\prime \prime 0+}$. It follows that

$$
\mathbf{h} \| \mathbf{j} \text { if } \operatorname{grad} T=0 .
$$

The two tensors are related by $\Sigma_{11}^{\prime 0+}=\Sigma_{11}^{\prime \prime 0+}$.

The form of even tensors describing effects occurring in an external magnetic field $\mathbf{H}$ are given in Fig. 2. The tensors of rank 3 describe effects linear in $\mathbf{H}$, those of rank 4 effects quadratic in $\mathbf{H}$.

In the special cases of cubic crystals (Laue classes 231' and $\left.4321^{\prime}\right)$ the Hall tensor $\rho_{i j l}^{-}$has only one independent element $\rho_{231}^{-}=\rho_{312}^{-}=\rho_{123}^{-}=-\rho_{321}^{-}=-\rho_{132}^{-}=-\rho_{213}^{-}$. The situation is

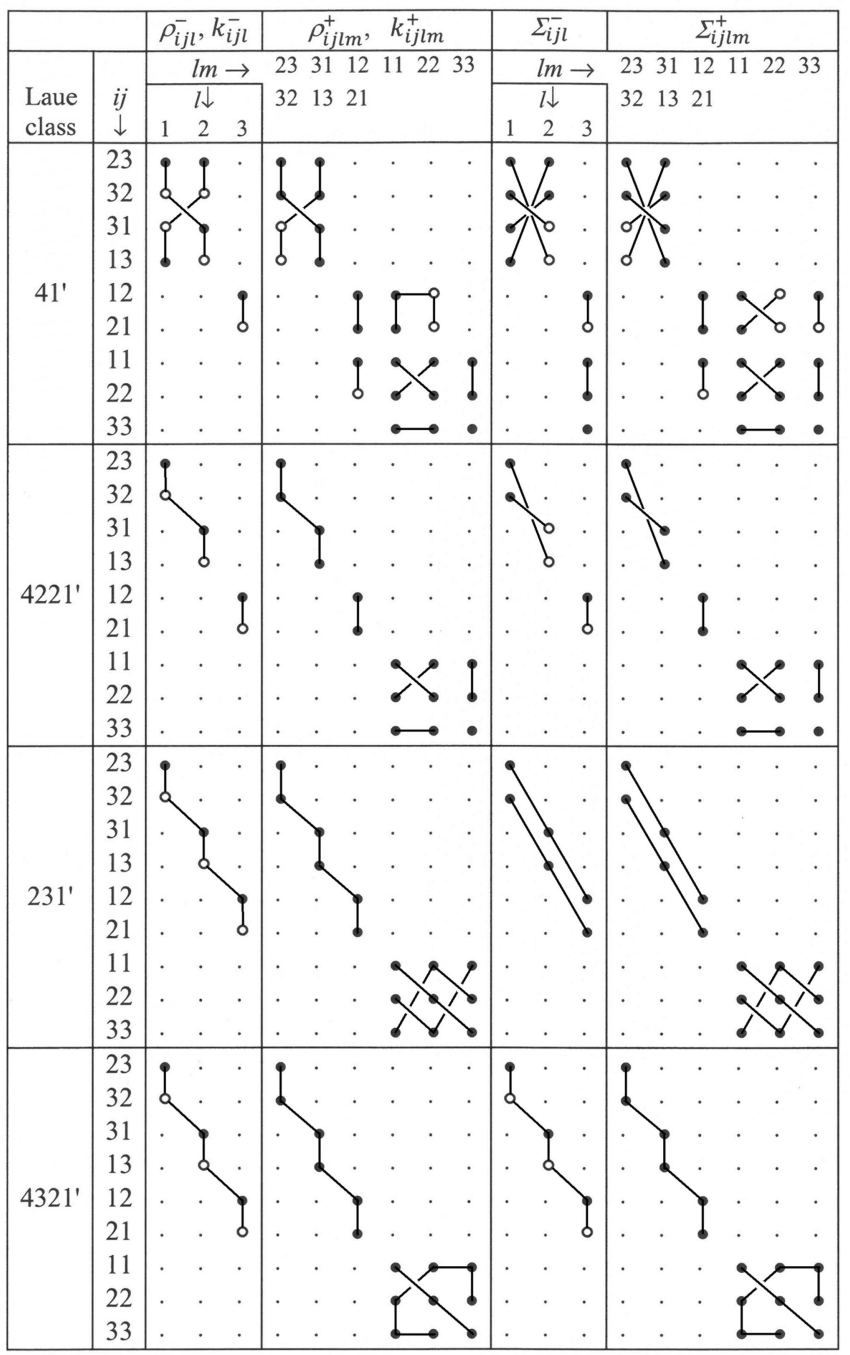

(b)
Figure 2 (continued)

(b) Tetragonal and cubic Laue classes. similar for the Righi-Leduc tensor. Numerical values for the Hall constant $R=\rho_{213}^{-}$have been given e.g. in Table 20.2 of Newnham (2005). For Laue class $4321^{\prime}$ the three independent components of $\rho_{i j l m}^{+}$, i.e. $\rho_{1111}^{+}, \rho_{1122}^{+}$and $\rho_{1212}^{+}$, give rise to longitudinal magnetoresistance, transverse magnetoresistance and the planar Hall effect, respectively. Similarly, the three independent components of $k_{i j l m}^{+}$, i.e. $k_{1111}^{+}, k_{1122}^{+}$and $k_{1212}^{+}$, give rise to longitudinal thermal magnetoresistance, transverse thermal magnetoresistance (also called the Maggi-RighiLeduc effect) and the planar thermal Hall effect, respectively (see ch. 20 in Newnham, 2005).

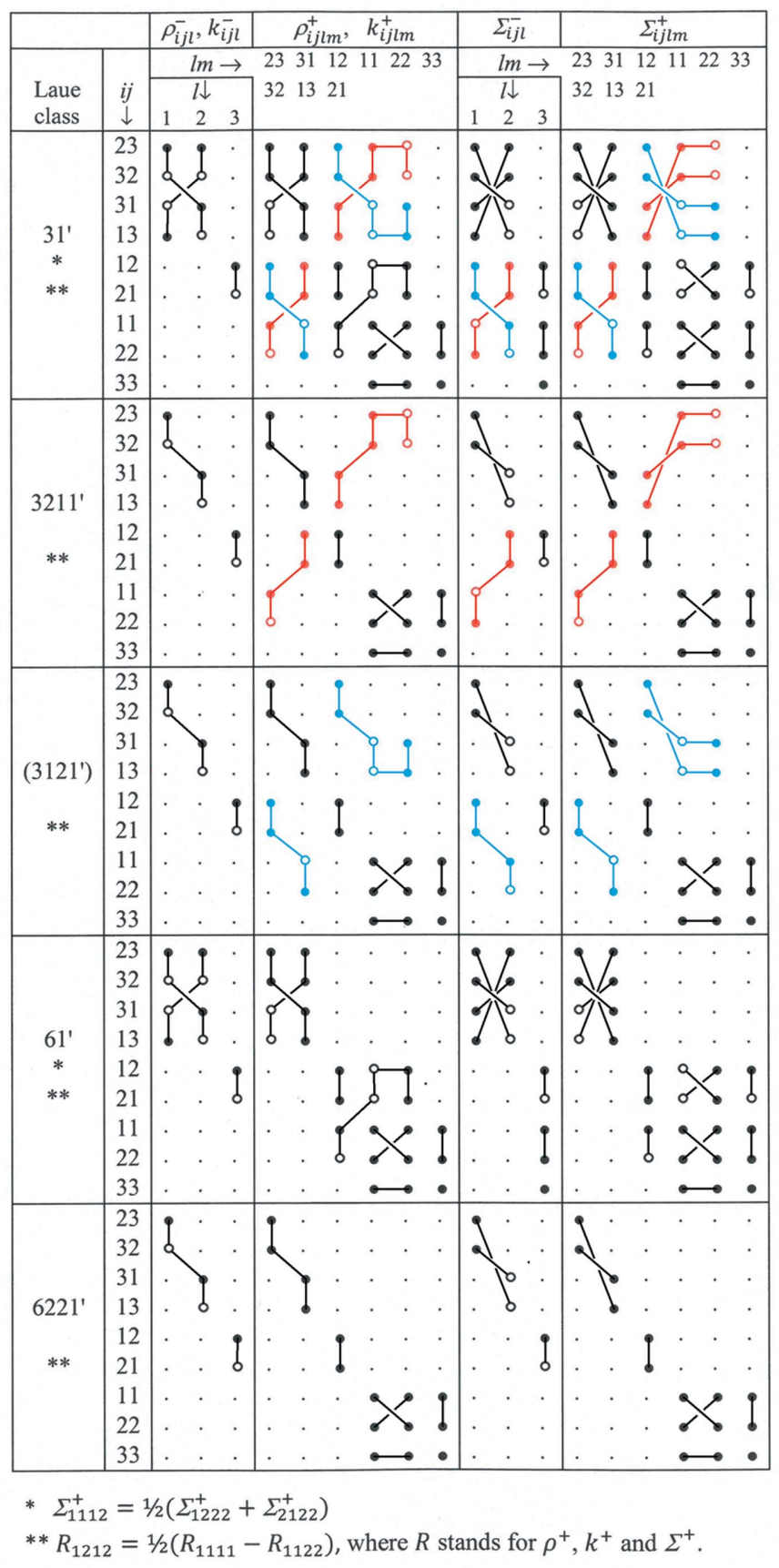

(c)

Figure 2 (continued)

(c) Trigonal and hexagonal Laue classes. 
Note in Fig. 2(c) that the property tensors $\rho_{i j k l}^{+}$and $k_{i j k l}^{+}$ satisfy an additional restriction, which reduces the number of independent components by 1 . Similarly, $\Sigma_{i j k l}^{+}$satisfies an additional restriction for the Laue classes $3211^{\prime},\left(3121^{\prime}\right), 6221^{\prime}$ and two additional restrictions for the Laue classes $31^{\prime}$ and $61^{\prime}$.

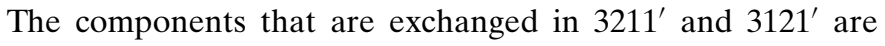
shown in different colours.

\subsection{Thermoelectric transport properties described by magnetic tensors}

For certain magnetically ordered crystals there may occur, in addition to the even property tensors, magnetic property tensors. Effects occurring in the absence of an external magnetic field, the spontaneous Hall effect $\rho^{0-}$ and spontaneous Righi-Leduc effect $k^{0-}$, are described by antisymmetric tensors of rank 2; the spontaneous Nernst effect $\Sigma^{\prime 0-}$ and spontaneous Ettingshausen effect $T \Sigma^{\prime \prime 0-}$ are described by general tensors of rank 2 , where $\Sigma_{i j}^{\prime \prime 0-}=-\Sigma_{j i}^{\prime 0-}$, as follows from equation (5). The form of these tensors is given in Fig. 3 for the point groups in category II.

\begin{tabular}{|c|c|c|c|}
\hline $\begin{array}{l}\text { Magnetic } \\
\text { form classes }\end{array}$ & & $\begin{array}{l}k_{i j}^{0-} \\
\rho_{i j}^{0-}\end{array}$ & $\Sigma_{i j}^{0-}$ \\
\hline & $\begin{array}{l}i \\
\downarrow\end{array}$ & $\begin{array}{ll} & j \downarrow \\
1 & 2 \\
1 & 3\end{array}$ & $\begin{array}{ccc} & & j \downarrow \\
& & \\
1 & 2 & 3\end{array}$ \\
\hline 1 & $\begin{array}{l}1 \\
2 \\
3\end{array}$ & & $\begin{array}{lll} & \bullet & \bullet \\
\bullet & \bullet & \bullet \\
\bullet & \bullet & \bullet\end{array}$ \\
\hline (211) & $\begin{array}{l}1 \\
2 \\
3\end{array}$ & & $\begin{array}{lll}\bullet & \cdot & \cdot \\
\cdot & \bullet & \bullet \\
. & \bullet & \bullet\end{array}$ \\
\hline 121 & $\begin{array}{l}1 \\
2 \\
3\end{array}$ & . & $\begin{array}{lll}\bullet & \cdot & \bullet \\
\cdot & \bullet & \cdot \\
\bullet & \cdot & \bullet\end{array}$ \\
\hline (112) & $\begin{array}{l}1 \\
2 \\
3\end{array}$ & . & $\begin{array}{lll} & \bullet & \cdot \\
\bullet & \bullet & \cdot \\
. & \cdot & \bullet\end{array}$ \\
\hline 222 & $\begin{array}{l}2 \\
3\end{array}$ & 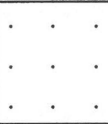 & $\begin{array}{lll} & \cdot & \cdot \\
\cdot & \bullet & \cdot \\
\cdot & \cdot & \bullet\end{array}$ \\
\hline $\begin{array}{l}3 \\
4 \\
6\end{array}$ & 1 & . & o \\
\hline $\begin{array}{l}321,(312) \\
422 \\
622\end{array}$ & $\begin{array}{l}1 \\
2\end{array}$ & $\begin{array}{lll}\cdot & \cdot & \cdot \\
\cdot & \cdot & . \\
\cdot & . & .\end{array}$ & 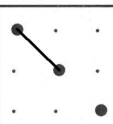 \\
\hline $\begin{array}{l}23 \\
432\end{array}$ & $\begin{array}{l}1 \\
2 \\
3\end{array}$ & $\begin{array}{lll}\cdot & \cdot & \cdot \\
\cdot & \cdot & \cdot \\
\cdot & \cdot & .\end{array}$ & i. \\
\hline
\end{tabular}

Figure 3

The restrictions for the point groups in category II satisfied by the property tensors of rank 2 listed on the right-hand side of Table 1 . The notation used for the matrix elements is explained at the bottom of Fig. 1. Magnetic form class symbols in parentheses refer to alternative choices of the Cartesian coordinate system.
Fig. 3 shows that there is no spontaneous Hall effect $\rho^{0-}$ and no spontaneous Righi-Leduc effect $k^{0-}$ for point groups with more than one rotation axis.

Let me show that the even and magnetic tensors can be determined individually by experiment.

If $\rho^{0}$ has been measured, the components $\rho_{i j}^{0+}$ of the electrical resistivity and the components $\rho_{i j}^{0-}$ of the spontaneous Hall effect are obtained as follows:

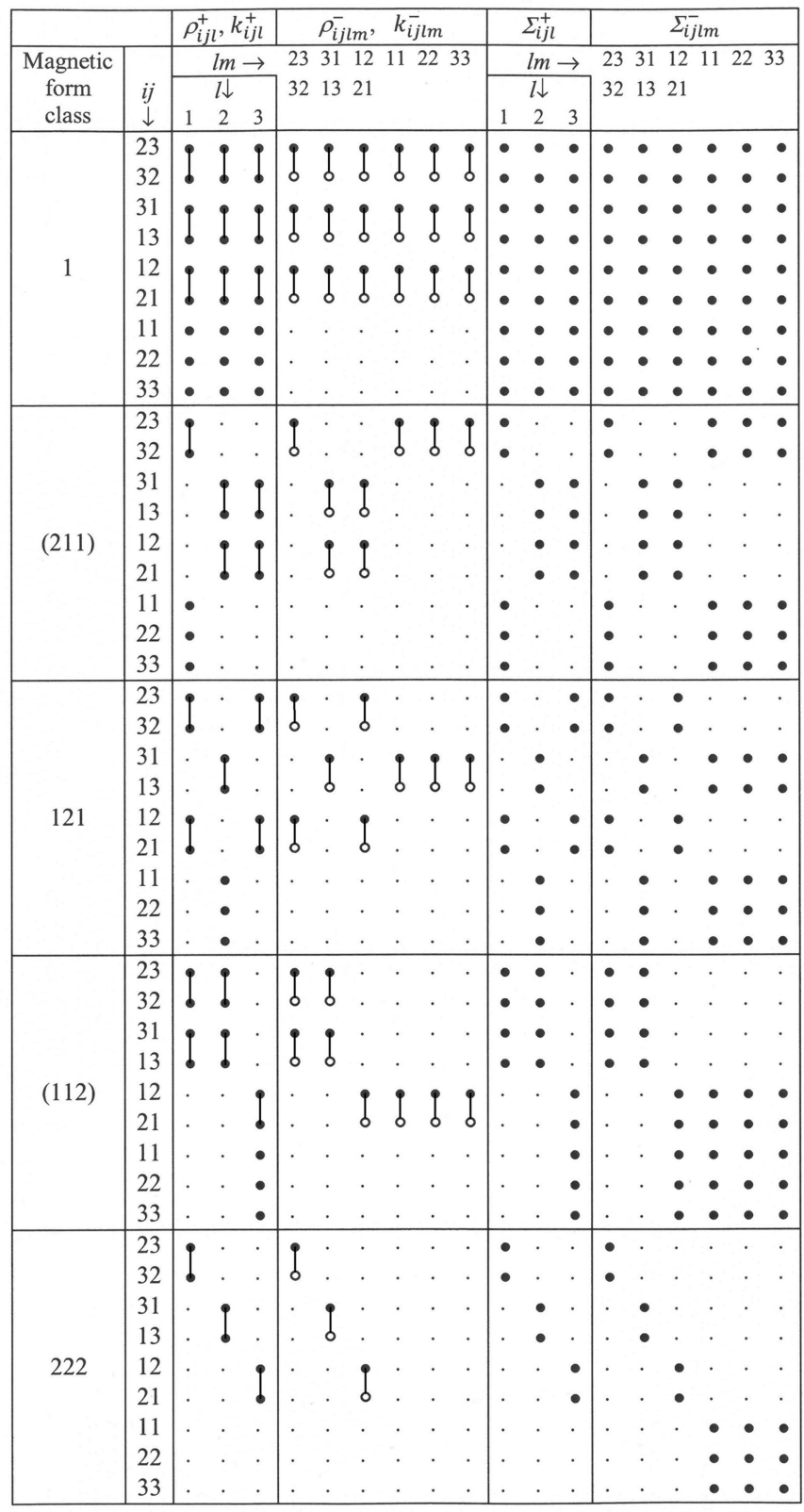

(a)

Figure 4

The restrictions for the point groups in category II satisfied by the property tensors of ranks 3 and 4 listed on the right-hand side of Table 1 . The notation used for the matrix elements is explained at the bottom of Fig. 1. Magnetic form class symbols in parentheses refer to alternative choices of the Cartesian coordinate system. (a) Anorthic, monoclinic and orthorhombic magnetic form classes in category II. 


$$
\begin{gathered}
\rho_{i j}^{0+}=\frac{1}{2}\left(\rho_{i j}^{0}+\rho_{j i}^{0}\right)=\rho_{j i}^{0+}, \\
\rho_{i j}^{0-}=\frac{1}{2}\left(\rho_{i j}^{0}-\rho_{j i}^{0}\right)=-\rho_{j i}^{0-} .
\end{gathered}
$$

Replacing $\rho$ by $k$, the analogous results for heat conductivity $k_{i j}^{0+}$ and the spontaneous Righi-Leduc effect $k_{i j}^{0-}$ are obtained.

If $\Sigma^{\prime 0}$ and $\Sigma^{\prime \prime 0}$ have been measured, the components $\Sigma_{i j}^{\prime 0+}$ of the Seebeck effect and the components $\Sigma_{i j}^{\prime 0-}$ of the spontaneous Nernst effect are obtained as follows:

$$
\begin{aligned}
& \Sigma_{i j}^{\prime 0+}=\frac{1}{2}\left(\Sigma_{i j}^{\prime 0}+\Sigma_{j i}^{\prime \prime 0}\right), \\
& \Sigma_{i j}^{\prime 0-}=\frac{1}{2}\left(\Sigma_{i j}^{\prime 0}-\Sigma_{j i}^{\prime \prime 0}\right) .
\end{aligned}
$$

Similarly, the components $\Sigma_{i j}^{\prime \prime 0+}$ of the Peltier effect and the components $\Sigma_{i j}^{\prime \prime 0-}$ of the spontaneous Ettingshausen effect are obtained as

$$
\Sigma_{i j}^{\prime \prime 0+}=\frac{1}{2}\left(\Sigma_{i j}^{\prime \prime 0}+\Sigma_{j i}^{\prime 0}\right)=\Sigma_{j i}^{\prime 0+},
$$

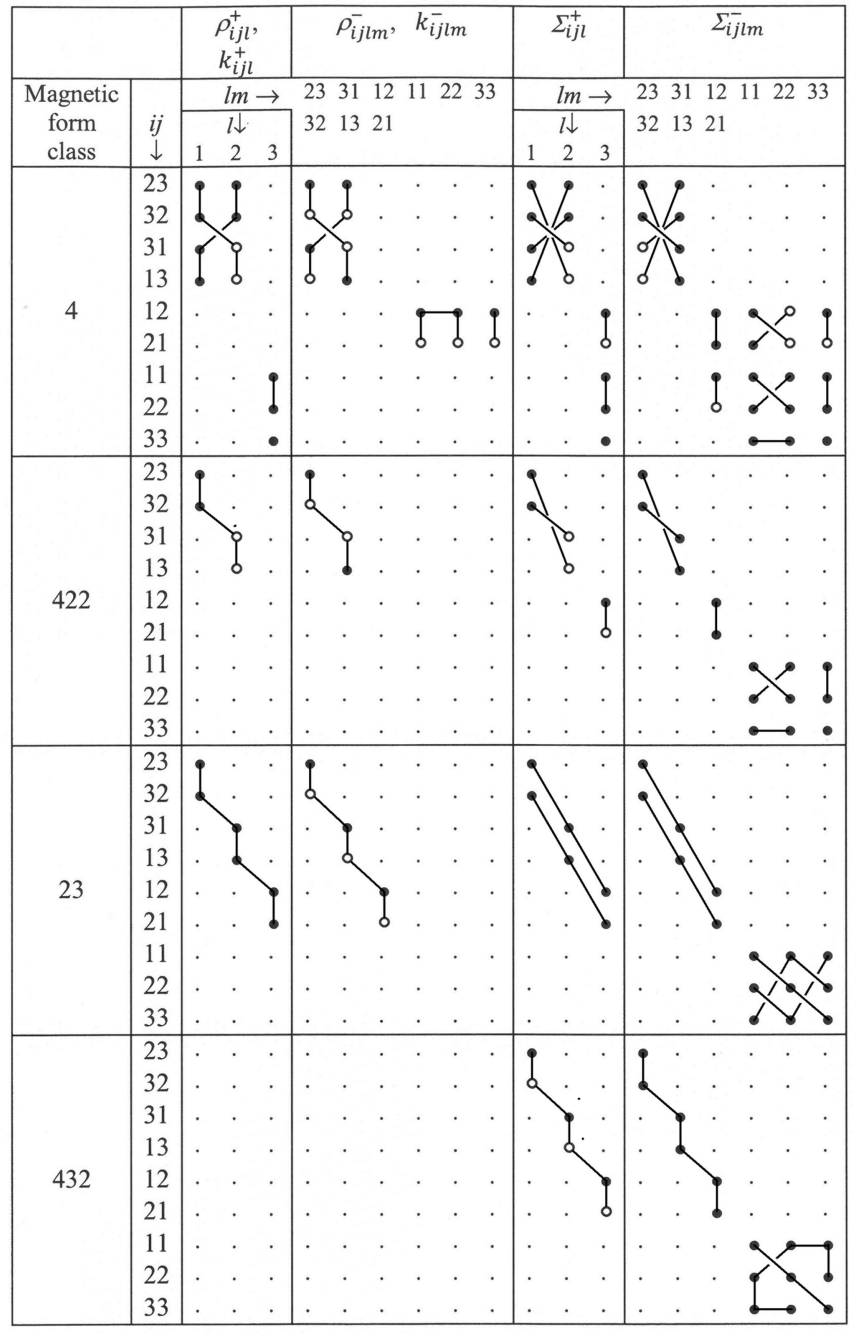

(b)

$$
\Sigma_{i j}^{\prime \prime 0-}=\frac{1}{2}\left(\Sigma_{i j}^{\prime \prime 0}-\Sigma_{j i}^{\prime 0}\right)=-\Sigma_{j i}^{\prime 0-} .
$$

The form of the even tensors has been given in Fig. 1, the form of the magnetic tensors in Fig. 3.

The tensors of rank 3 describing effects linear in $\mathbf{H}$ and the tensors of rank 4 describing effects quadratic in $\mathbf{H}$ can similarly be split into an even and a magnetic tensor.

The form of magnetic tensors describing effects occurring in an external magnetic field $\mathbf{H}$ are given in Fig. 4 for the point groups in category II.

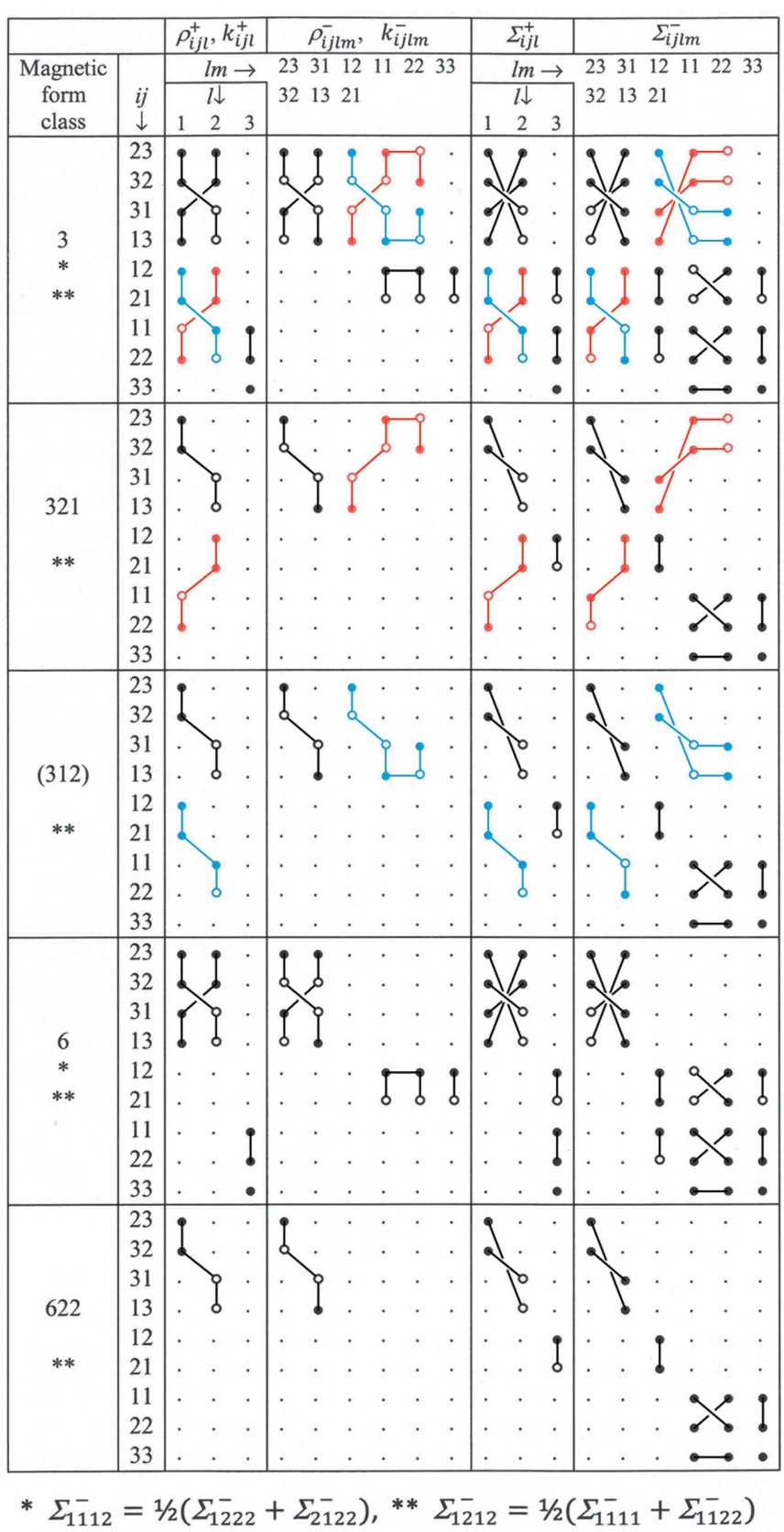

(c)

Figure 4 (continued)

(c) Trigonal and hexagonal magnetic form classes in category II. 
We now deal with the magnetic form classes in category III $^{a 1}$. The form of the property tensors of rank 2 is given in Fig. 5.

Fe (body-centred cubic), Co (hexagonal close-packed) and $\mathrm{Ni}$ (face-centred cubic) have in the paramagnetic phase (i.e. above the Curie point) point groups $m \overline{3} m 1^{\prime}, 6 / m m m 1^{\prime}$ and $m \overline{3} m 1^{\prime}$, respectively; in the ferromagnetic phase they have point groups $4 / \mathrm{mm}^{\prime} \mathrm{m}^{\prime}, 6 / \mathrm{mm}^{\prime} \mathrm{m}^{\prime}$ and $\overline{3} \mathrm{~m}^{\prime}$ with spontaneous magnetization parallel to the principal symmetry axis [see Table 16.3 and Fig. 16.8 in Newnham (2005)]. Fig. 5 shows that the corresponding magnetic form classes $42^{\prime} 2^{\prime}, 62^{\prime} 2^{\prime}$ and $32^{\prime}$ have only one independent component describing the spontaneous Hall effect, and similarly for the three other sponta-

\begin{tabular}{|c|c|c|c|}
\hline $\begin{array}{l}\text { Magnetic } \\
\text { form classes }\end{array}$ & & $\begin{array}{l}k_{i j}^{0-} \\
\rho_{i j}^{0-} \\
\end{array}$ & $\Sigma_{i j}^{0-}$ \\
\hline & $\begin{array}{l}i \\
\downarrow\end{array}$ & \begin{tabular}{|ccc} 
& $j \downarrow$ & \\
& & \\
1 & 2 & 3
\end{tabular} & 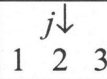 \\
\hline$\left(2^{\prime} 11\right)$ & $\begin{array}{l}1 \\
2 \\
3\end{array}$ & of. & $\begin{array}{lll} & \bullet & \bullet \\
\bullet & \cdot & \cdot \\
\bullet & \cdot & \cdot\end{array}$ \\
\hline $12^{\prime} 1$ & \begin{tabular}{|l|}
1 \\
2 \\
3 \\
\end{tabular} & & $\begin{array}{lll}\cdot & \bullet & \cdot \\
\bullet & \cdot & \bullet \\
\cdot & \bullet & \cdot \\
\end{array}$ \\
\hline$\left(112^{\prime}\right)$ & \begin{tabular}{|l|}
1 \\
2 \\
3
\end{tabular} & & $\begin{array}{lll}\cdot & \cdot & \bullet \\
\cdot & \cdot & \bullet \\
\bullet & \bullet & \cdot\end{array}$ \\
\hline$\left(22^{\prime} 2^{\prime}\right)$ & $\begin{array}{l}1 \\
2 \\
3\end{array}$ & & $\begin{array}{lll}\cdot & \cdot & \cdot \\
\cdot & \cdot & \bullet \\
\cdot & \bullet & .\end{array}$ \\
\hline$\left(2^{\prime} 22^{\prime}\right)$ & \begin{tabular}{|l|}
1 \\
2 \\
3 \\
\end{tabular} & & $\begin{array}{lll}\cdot & \cdot & \bullet \\
\cdot & \cdot & . \\
\bullet & \cdot & . \\
\end{array}$ \\
\hline $2^{\prime} 2^{\prime} 2$ & $\begin{array}{l}1 \\
2 \\
3\end{array}$ & . & $\begin{array}{lll} & \bullet & \\
\bullet & \cdot & . \\
\cdot & \cdot & . \\
. & \cdot & .\end{array}$ \\
\hline $4^{\prime}$ & \begin{tabular}{|l|}
1 \\
2 \\
3 \\
\end{tabular} & 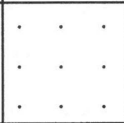 & 8 \\
\hline $4^{\prime} 22^{\prime}$ & \begin{tabular}{|l|}
1 \\
2 \\
3 \\
\end{tabular} & 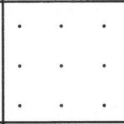 & . \\
\hline$\left(4^{\prime} 2^{\prime} 2\right)$ & \begin{tabular}{|l|}
1 \\
2 \\
3 \\
\end{tabular} & 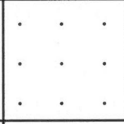 & . \\
\hline $\begin{array}{l}32^{\prime} 1,\left(312^{\prime}\right) \\
42^{\prime} 2^{\prime} \\
62^{\prime} 2^{\prime}\end{array}$ & \begin{tabular}{|l|}
1 \\
2 \\
3 \\
\end{tabular} & . & \% \\
\hline $\begin{array}{l}6^{\prime} \\
6 ' 22^{\prime},\left(6^{\prime} 2^{\prime} 2\right) \\
4^{\prime} 32^{\prime}\end{array}$ & \begin{tabular}{|l|}
1 \\
2 \\
3
\end{tabular} & 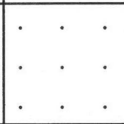 & $\begin{array}{lll} & \cdot & . \\
. & \cdot & . \\
. & . & .\end{array}$ \\
\hline
\end{tabular}

Figure 5

The restrictions for the point groups in category $\mathrm{III}^{a 1}$ satisfied by the property tensors of rank 2 listed on the right-hand side of Table 1 . The notation used for the matrix elements is explained at the bottom of Fig. 1. Magnetic form class symbols in parentheses refer to alternative choices of the Cartesian coordinate system. neous effects. Numerical values of this component for $\mathrm{Fe}, \mathrm{Cu}$ and $\mathrm{Ni}$ have been given for the spontaneous Hall effect by Miyasato et al. (2007), Nagaosa et al. (2010) and Ködderitzsch

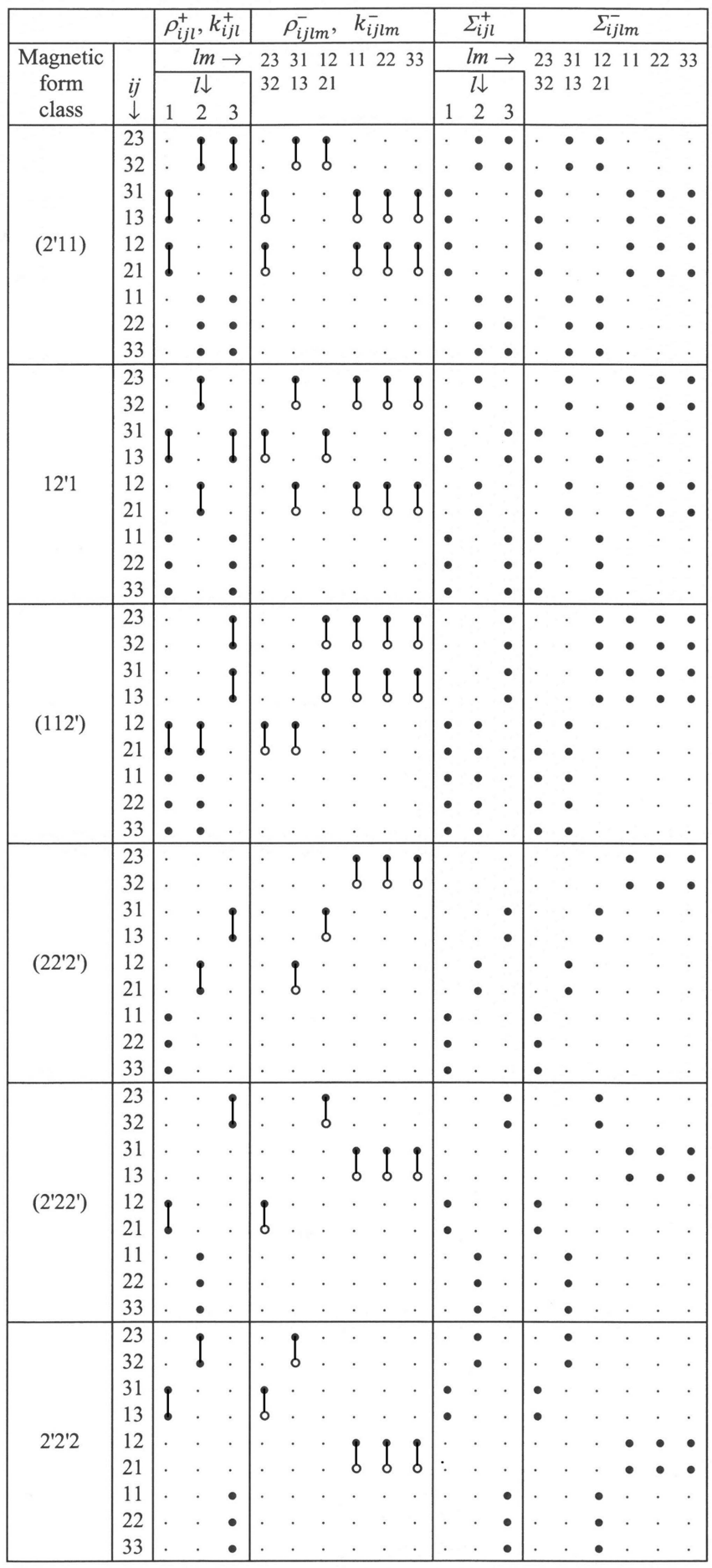

(a)

Figure 6

The restrictions for the point groups in category III $^{a 1}$ satisfied by the property tensors of ranks 3 and 4 listed on the right-hand side of Table 1. The notation used for the matrix elements is explained at the bottom of Fig. 1. Magnetic form class symbols in parentheses refer to alternative choices of the Cartesian coordinate system. (a) Anorthic, monoclinic and orthorhombic magnetic form classes in category III $^{a 1}$. 
et al. (2015), and for the spontaneous Nernst effect by Miyasato et al. (2007).

The form of magnetic tensors describing effects occurring in an external magnetic field $\mathbf{H}$ is given in Fig. 6 for the point groups in category III $^{a 1}$. The tensors of rank 3 describe effects linear in $\mathbf{H}$, the tensors of rank 4 describe effects quadratic in H.

For category III ${ }^{a 1}$, the experimental separation of the even and magnetic property tensors is particularly easy because for standard coordinate systems each non-zero component is due either exclusively to the even or exclusively to the magnetic tensor, with the exception of the magnetic form classes $4^{\prime}, 4^{\prime} 22^{\prime}$ and $4^{\prime} 32^{\prime}$.

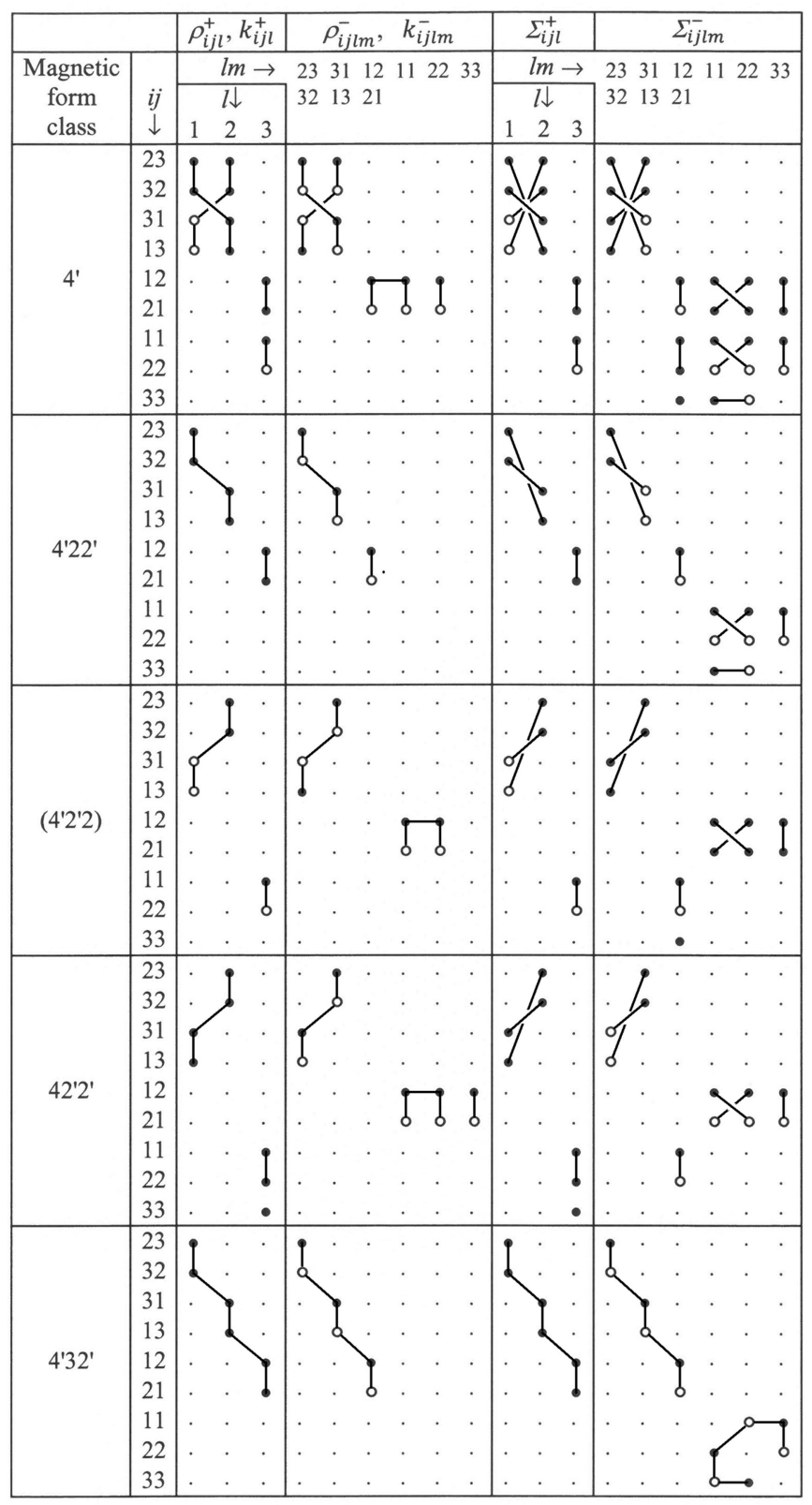

(b)

Figure 6 (continued)

(b) Tetragonal and cubic magnetic form classes in category III $^{a 1}$.

\section{Remarks and corrections to the literature}

The forms of $\left(\rho^{0+}, k^{0+}\right)$ and $\Sigma^{\prime \prime 0+}$ given in Fig. 1 correspond to those given for $\boldsymbol{\sigma}$ and $\boldsymbol{\tau}^{\prime}$ in Table V of Kleiner (1966); the form of $\Sigma^{\prime 0+}$ corresponds to the form of $\tau$ given in his Table IV. A

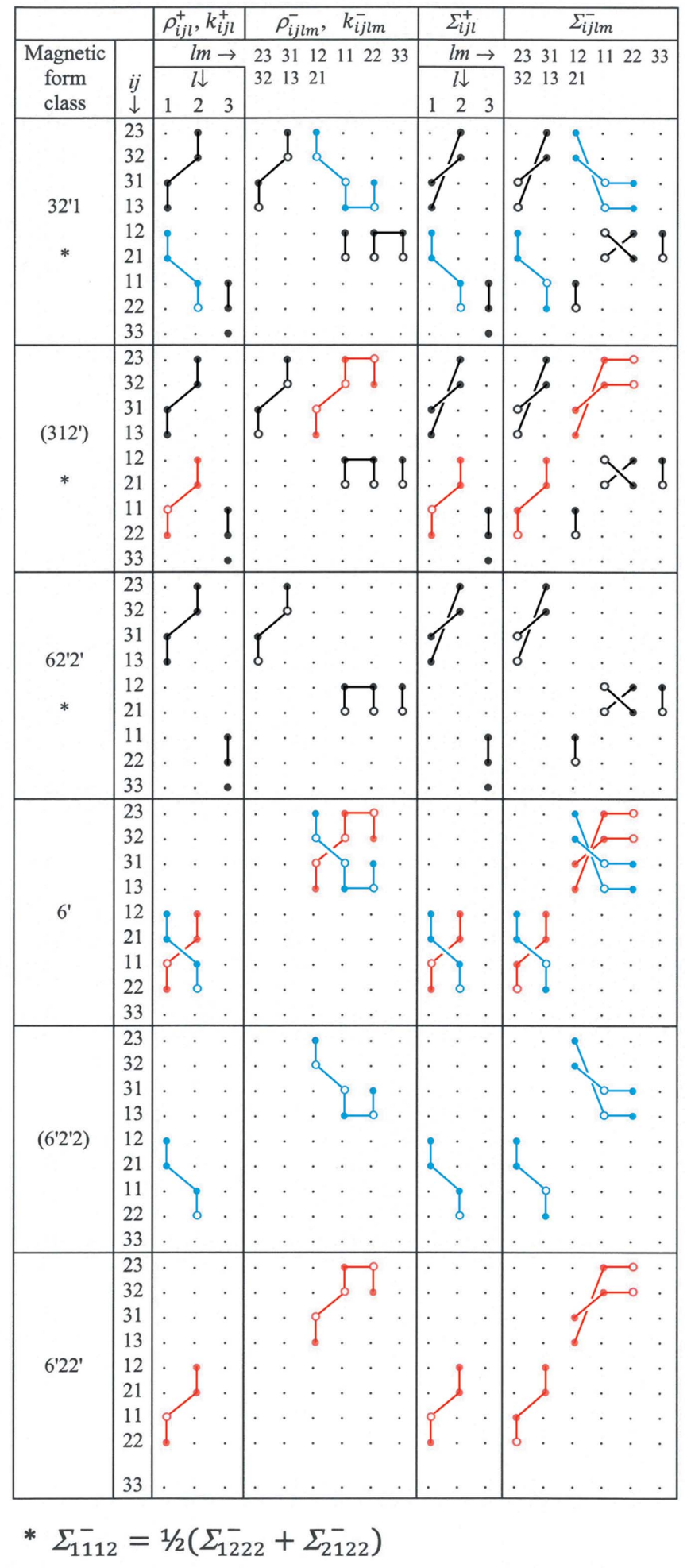

(c)

Figure 6 (continued)

(c) Trigonal and hexagonal magnetic form classes in category $\mathrm{III}^{a 1}$. 
comparison shows that the class $21^{\prime}$ in his Table $\mathrm{V}$ corresponds to our $\left(1121^{\prime}\right)$, i.e. the monoclinic axis has been chosen along $z$. Note also that wrong results are given in Table $\mathrm{V}$ for the form of $\tau^{\prime}$ in the Laue classes $3^{\prime}\left(=31^{\prime}\right), 41^{\prime}, 61^{\prime}$ and the limiting class $\infty 1^{\prime}$.

Instead of the magnetic tensor $\rho^{0-}$, Kleiner (1966) considers the sum $\rho^{0}=\rho^{0+}+\rho^{0-}$, and similarly in the three other cases. For the point groups in category II he finds that $\sigma=\rho^{0+}+\rho^{0-}$ (or $k^{0+}+k^{0-}$ ), $\tau=\Sigma^{\prime 0+}+\Sigma^{\prime 0-}$ and $\tau^{\prime}=\Sigma^{\prime \prime 0+}+\Sigma^{\prime \prime 0-}$ all have the form of $\boldsymbol{\tau}$ given in his Table IV, i.e. $\boldsymbol{\sigma}, \boldsymbol{\tau}$ and $\boldsymbol{\tau}^{\prime}$ are independent tensors of rank 2 without internal symmetry; we obtained in Figs. 1 and 3 that $\rho^{0+}$ and $k^{0+}$ are symmetric, $\rho^{0-}$ and $k^{0-}$ antisymmetric in their two indices and that $\Sigma_{i j}^{\prime \prime 0+}=\Sigma_{j i}^{\prime 0+}, \Sigma_{i j}^{\prime \prime 0-}=-\Sigma_{j i}^{\prime 0-}$.

Comparing $\Sigma_{i j l}^{-}$in Fig. 2 with $\sigma$ in Table VII of Seemann et al. (2015), we find that the same components are zero and the same components are equal or opposite, except that the results for $3211^{\prime}$ and $3121^{\prime}$ are interchanged. This tells us that Seemann et al. (2015) do not adhere to the usual convention concerning the orientation of the Cartesian coordinate system in the case of the hexagonal crystal family. The components that are exchanged in $3211^{\prime}$ and $3121^{\prime}$ are shown in different colours in Fig. 2(c).

Malinowski (1986) considers the Hall tensor and presents in his Table IA results related to the results given for $\rho_{i j l}^{-}$and $\rho_{i j l}^{+}$ in our Figs. 2, 4 and 6. Similarly as Kleiner (1966) and Seemann et al. (2015) do for tensors of rank 2, he does not separate the magnetic tensor $\rho_{i j l}^{+}$(which describes linear magnetoresistance) and the even tensor $\rho_{i j l}^{-}$(which describes the ordinary Hall effect). His results generally agree with ours; however, a number of mistakes should be noted in his Table IA: in the fourth entry on p. 37 (i.e. K: 4, ..) $P_{321}$ in the second row of the matrix should be replaced by $P_{231}$. Five of the 122 space-time point groups, i.e. $4 / m^{\prime}, 4^{\prime} / m^{\prime}, \overline{3}^{\prime}, 6 / m^{\prime}$ and $6^{\prime} / m$ are missing in Table IA. For these five groups, $\sigma$ has the form given in the fourth entry on p. 38 (i.e. $\mathrm{K}: 41^{\prime}, \ldots$ ). Note that ' 32 (when $2 \| x$ ), $3 m, \overline{3} m$ (when $m \perp x$ )' in Table IA corresponds to our magnetic form class 321. Similarly ' 32 (when $2 \| y$ ), $3 m, \overline{3} m$ (when $m \perp y$ )' corresponds to our (312), ' ' $\overline{6}^{\prime} m 2^{\prime}$, $\overline{6}^{\prime} m^{\prime} 2,6^{\prime} 2^{\prime} 2,6^{\prime} m^{\prime} m, 6^{\prime} / m^{\prime} m^{\prime} m$ (when $2 \| x$ or $m \perp x$ )' corresponds to $6^{\prime} 22^{\prime}$, and ' $\bar{\sigma}^{\prime} m 2^{\prime}, \overline{6}^{\prime} m^{\prime} 2,6^{\prime} 2^{\prime} 2,6^{\prime} m^{\prime} m, 6^{\prime} / m^{\prime} m^{\prime} m$ (when $2 \| y$ or $m \perp y)^{\prime}$ corresponds to $\left(6^{\prime} 2^{\prime} 2\right)$.

It follows from the results in Fig. 2 that the column $\rho_{i j k l}$ in Table 20.1 of Newnham (2005) should be corrected as follows: $45(12)$ in the row for Laue class 3, 25(8) in the rows for 32 and 6. Similarly, in his Table 21.5 the column $\alpha_{m n p q}$ should be corrected as follows: 27(12) in the row for Laue class 6 and $15(5)$ in the row for 23; also column $\alpha_{m n p}$ needs a correction: $6(2)$ in the row for 23.

\section{Summary}

Taking also magnetic order into account, crystals can be assigned to one of 122 classes according to their space-time point-group symmetry. For all these classes, we derived the restrictions following from the Neumann principle on the form of even and of magnetic tensors of ranks 2, 3 and 4 describing thermoelectric transport properties independent, linear and quadratic in an external magnetic field $\mathbf{H}$, respectively. The results have been illustrated with experimental values of tensor components.

A number of errors in the literature concerning the form of tensors describing thermoelectric transport properties have been corrected.

Kleiner (1966), Malinowski (1986), Seemann et al. (2015) and the Bilbao Crystallographic Server (2017) consider the restrictions on the sum of an even tensor occurring in crystals belonging to any of the 122 space-time point groups and a magnetic tensor occurring only in crystals belonging to point groups allowing certain types of magnetic order, as indicated in Table 3. Similarly, Wimmer et al. (2016) consider the restrictions on the sum of an electric and a magnetoelectric property tensor in their consideration of spin-orbit torques.

The main message of the present paper is the proof that for each of the pairs given in the 12 rows of Table 1 , the even and the magnetic tensor can be determined individually by experiment. The thermoelectric tensors $\Sigma^{\prime}$ and $\Sigma^{\prime \prime}$ are related differently for even and magnetic tensors; the electric and thermal tensors, $\rho$ and $k$, have different internal symmetries for even and magnetic tensors.

In other words, treating the even and magnetic tensors as distinct tensors means:

(i) Making it possible (as in the case of tensors describing equilibrium properties) to associate to each property tensor one of the characteristics even, magnetic, electric or magnetoelectric, describing its behaviour under inversions.

(ii) Distinguishing the energy dissipative and the lossless part of $\rho$.

(iii) Distinguishing between electrical resistivity and the spontaneous Hall effect and, similarly, distinguishing between the two effects in each of the other 11 rows of Table 1.

(iv) Distinguishing between the tensors related by $\Sigma_{i j}^{\prime \prime 0}=\Sigma_{j i}^{\prime 0}$ and those related by $\Sigma_{i j}^{\prime \prime 0}=-\Sigma_{j i}^{\prime 0}$, between the tensors related by $\Sigma_{i j l}^{\prime \prime}=\Sigma_{j i l}^{\prime}$ and those related by $\Sigma_{i j l}^{\prime \prime}=-\Sigma_{j i l}^{\prime}$, between the tensors related by $\Sigma_{i j l m}^{\prime \prime}=\Sigma_{j i l m}^{\prime}$ and those related by $\Sigma_{i j l m}^{\prime \prime}=-\Sigma_{j i l m}^{\prime}$.

\section{References}

Bilbao Crystallographic Server (2017). MTENSOR: symmetryadapted form of crystal tensors in magnetic phases. http:// www.cryst.ehu.es.

Borovik-Romanov, A. S., Grimmer, H. \& Kenzelmann, M. (2014). In International Tables for Crystallography, Vol. D, Physical Properties of Crystals, 2nd ed., edited by A. Authier. Chichester: Wiley.

Butzal, H. D. \& Birss, R. R. (1982). Physica (Utrecht) A, 114, 518-521. Grimmer, H. (1991). Acta Cryst. A47, 226-232.

Grimmer, H. (1993). Acta Cryst. A49, 763-771.

Grimmer, H. (1994). Ferroelectrics, 161, 181-189.

IEEE Standard on Piezoelectricity (1988). ANSI/IEEE Std 176-1987. New York: IEEE.

International Tables for Crystallography (2016). Vol. A, Space-Group Symmetry, 6th ed., edited by M. I. Aroyo. Chichester: Wiley.

Jahn, H. A. (1949). Acta Cryst. 2, 30-33.

Kleiner, W. H. (1966). Phys. Rev. 142, 318-326. 
Ködderitzsch, D., Chadova, K. \& Ebert, H. (2015). Phys. Rev. B, 92, 184415.

Landau, L. D. \& Lifshitz, E. M. (1958). Statistical Physics, section 119. London: Pergamon Press.

Malinowski, S. (1986). Acta Phys. Pol. A, 69, 33-43.

Miyasato, T., Abe, N., Fujii, T., Asamitsu, A., Onoda, S., Onose, Y., Nagaosa, N. \& Tokura, Y. (2007). Phys. Rev. Lett. 99, 086602.

Nagaosa, N., Sinova, J., Onoda, S., MacDonald, A. H. \& Ong, N. P. (2010). Rev. Mod. Phys. 82, 1539-1592.

Newnham, R. E. (2005). Properties of Materials. Oxford University Press.

Nye, J. F. (1985). Physical Properties of Crystals, 2nd ed. Oxford: Clarendon Press.
Schmid, H. (1973). Int. J. Magn. 4, 337-361.

Seemann, M., Ködderitzsch, D., Wimmer, S. \& Ebert, H. (2015). Phys. Rev. B, 92, 155138 .

Shtrikman, S. \& Thomas, H. (1965a). Solid State Commun. 3, 147-150.

Shtrikman, S. \& Thomas, H. (1965b). Solid State Commun. 3, civ.

Sirotin, Yu. I. \& Shaskolskaya, M. P. (1982). Fundamentals of Crystal Physics. Moscow: Mir Publishers.

Vainshtein, B. K. (1996). Fundamentals of Crystals: Symmetry and Methods of Structural Crystallography. Berlin: Springer-Verlag.

White, G. K. \& Minges, M. L. (1997). Int. J. Thermophys. 18, 1269 1327.

Wimmer, S., Chadova, K., Seemann, M., Ködderitzsch, D. \& Ebert, H. (2016). Phys. Rev. B, 94, 054415. 